features have been obtained, see: Ahlers, Temme, Erker,

\section{Crystal structure of bis $\left(\eta^{5}\right.$-cyclopenta- dienyl)(1,4-di-tert-butylbuta-1-en-3-yn-1- yl)zirconium(IV) $\mu_{2}$-hydroxido-bis[tris- (pentafluorophenyl)borate]}

\author{
Vladimir V. Burlakov, ${ }^{\text {a }}$ Anke Spannenberg, ${ }^{\text {b }}$ Perdita \\ Arndt $^{\mathrm{b}}$ and Uwe Rosenthal ${ }^{\mathrm{b}_{*}}$

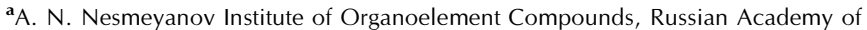 \\ Sciences, Vavilov St. 28, 119991 Moscow, Russian Federation, and ${ }^{\mathbf{b}}$ Leibniz-Institut \\ für Katalyse e. V. an der Universität Rostock, Albert-Einstein-Strasse 29a, 18059 \\ Rostock, Germany. *Correspondence e-mail: uwe.rosenthal@catalysis.de
}

Received 15 December 2014; accepted 23 February 2015

Edited by M. Nieger, University of Helsinki, Finland

Alkyl zirconocene cations have been of considerable interest as reactive species in many polymerization processes. In the crystal structure of the title compound, $\left[\mathrm{Zr}\left(\mathrm{C}_{12} \mathrm{H}_{19}\right)\left(\mathrm{C}_{5} \mathrm{H}_{5}\right)_{2}\right]\left(\mathrm{C}_{36} \mathrm{HB}_{2} \mathrm{~F}_{30} \mathrm{O}\right)$, the $\left[\mathrm{Zr}\left(\mathrm{C}_{5} \mathrm{H}_{5}\right)_{2}((t-\mathrm{Bu})-\right.$ $\left.\left.\mathrm{C}=\mathrm{C}(\mathrm{H})-\mathrm{C}_{2}(t-\mathrm{Bu})\right)\right]^{+}$cation displays a buta-1-en-3-yne ligand side-on coordinated to a typical bent zirconocene [centroid $(\mathrm{cp})-\mathrm{Zr}-$ centroid $(\mathrm{cp})=131.4(3)^{\circ}, \mathrm{Zr}-\mathrm{C}$ (buta-1en-3-yne) $=2.255(3), 2.597(3)$ and $2.452(2) \AA]$. In the $\left[\mathrm{HO}\left(\mathrm{B}\left(\mathrm{C}_{6} \mathrm{~F}_{5}\right)_{3}\right)_{2}\right]^{-}$anion, intramolecular $\mathrm{O}-\mathrm{H} \cdots \mathrm{F}$ hydrogen bonds are observed. One tert-butyl group in the complex cation is disordered over two sets of sites with occupancies 0.701(4):0.299(4).

Keywords: crystal structure; zirconocene; buta-1-en-3-yne; borate anion; intramolecular $\mathrm{O}-\mathrm{H} \cdots \mathrm{F}$ hydrogen bonds.

CCDC reference: 1050794

\section{Related literature}

For examples of the coordination of a buta-en-yne ligand to a group IV transition metal atom, see: Erker et al. (2004); Ahlers, Temme, Erker, Fröhlich \& Fox (1997). For complexation of a buta-1-en-3-yne as a bridging ligand between two metallocenes, see: Ahlers, Temme, Erker, Fröhlich \& Zippel (1997); Burlakov et al. (2010). For an example of the structure of the hydroxyl borate anion and its formation, see: Liptau et al. (2004). Stoichimetric reactions of alkylzirconocene complexes with $\mathrm{B}\left(\mathrm{C}_{6} \mathrm{~F}_{5}\right)_{3}$ have been investigated and different reaction modes (e.g. $\mathrm{C}-\mathrm{C}$ bond coupling or cleavage) and compounds exhibiting interesting structural

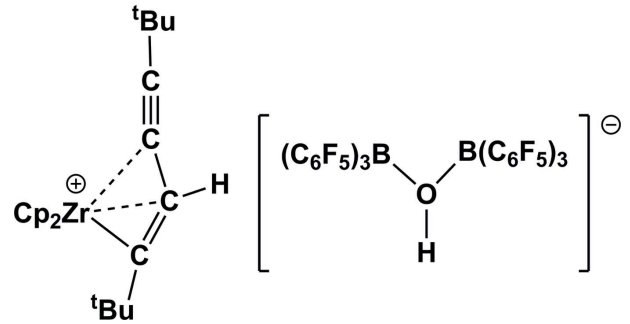

2. Experimental

\subsection{Crystal data}

$\left[\mathrm{Zr}\left(\mathrm{C}_{12} \mathrm{H}_{19}\right)\left(\mathrm{C}_{5} \mathrm{H}_{5}\right)_{2}\right]\left(\mathrm{C}_{36} \mathrm{HB}_{2} \mathrm{~F}_{30} \mathrm{O}\right)$

$M_{r}=1425.66$

Triclinic, $P \overline{1}$

$a=12.8896(5) \AA$

$b=13.6334(5) \AA$

$c=16.5466(6) \AA$

$\alpha=86.730$ (3)

$\beta=75.389$ (3)

\subsection{Data collection}

Stoe IPDS II diffractometer

Absorption correction: numerical ( $X$-SHAPE and $X$-RED32; Stoe $\&$ Cie, 2005)

$T_{\min }=0.810, T_{\max }=0.966$

\subsection{Refinement}

$R\left[F^{2}>2 \sigma\left(F^{2}\right)\right]=0.037$

$w R\left(F^{2}\right)=0.083$

$S=0.84$

13119 reflections

820 parameters

27 restraints

$$
\begin{aligned}
& \gamma=77.581(3)^{\circ} \\
& V=2747.84(18) \AA^{3} \\
& Z=2 \\
& \text { Mo K } \alpha \text { radiation } \\
& \mu=0.35 \mathrm{~mm}^{-1} \\
& T=200 \mathrm{~K} \\
& 0.25 \times 0.25 \times 0.17 \mathrm{~mm}
\end{aligned}
$$

47172 measured reflections 13119 independent reflections 8407 reflections with $I>2 \sigma(I)$ $R_{\text {int }}=0.040$
Table 1

Hydrogen-bond geometry $\left(\AA{ }^{\circ}\right)$.

\begin{tabular}{lllll}
\hline$D-\mathrm{H} \cdots A$ & $D-\mathrm{H}$ & $\mathrm{H} \cdots A$ & $D \cdots A$ & $D-\mathrm{H} \cdots A$ \\
\hline $\mathrm{O} 1-\mathrm{H} 1 \cdots \mathrm{F} 1$ & $0.76(3)$ & $2.10(3)$ & $2.722(2)$ & $139(2)$ \\
$\mathrm{O} 1-\mathrm{H} 1 \cdots \mathrm{F} 30$ & $0.76(3)$ & $2.08(3)$ & $2.723(2)$ & $142(2)$ \\
\hline
\end{tabular}

Data collection: $X$-AREA (Stoe \& Cie, 2005); cell refinement: $X$ $A R E A$; data reduction: $X$-AREA; program(s) used to solve structure: SHELXS97 (Sheldrick, 2008); program(s) used to refine structure: SHELXL2014 (Sheldrick, 2015); molecular graphics: $X P$ in SHELXTL (Sheldrick, 2008); software used to prepare material for publication: SHELXL2014.

\section{Acknowledgements}

We would like to thank our technical and analytical staff for assistance. Financial support by the Deutsche Forschungsgemeinschaft (RO 1269/9-1) and the Russian Foundation for 
Basic Research (project code 15-03-03485) is gratefully acknowledged.

Supporting information for this paper is available from the IUCr electronic archives (Reference: NR2057).

\section{References}

Ahlers, W., Temme, B., Erker, G., Fröhlich, R. \& Fox, T. (1997). J. Organomet. Chem. 527, 191-201.

Ahlers, W., Temme, B., Erker, G., Fröhlich, R. \& Zippel, F. (1997). Organometallics, 16, 1440-1444.
Burlakov, V. V., Arndt, P., Baumann, W., Spannenberg, A. \& Rosenthal, U. (2004). Organometallics, 23, 5188-5192.

Burlakov, V. V., Beweries, T., Kaleta, K., Bogdanov, V. S., Arndt, P., Baumann, W., Spannenberg, A., Shur, V. B. \& Rosenthal, U. (2010). Organometallics, 29, 2367-2371.

Erker, G., Venne-Dunker, S., Kehr, G., Kleigrewe, N., Fröhlich, R., MückLichtenfeld, C. \& Grimme, S. (2004). Organometallics, 23, 4391-4395.

Liptau, P., Neumann, M., Erker, G., Kehr, G., Fröhlich, R. \& Grimme, S. (2004). Organometallics, 23, 21-25.

Sheldrick, G. M. (2008). Acta Cryst. A64, 112-122.

Sheldrick, G. M. (2015). Acta Cryst. C71, 3-8.

Stoe \& Cie (2005). X-AREA, X-RED32 and X-SHAPE. Stoe \& Cie, Darmstadt, Germany. 


\section{supporting information}

Acta Cryst. (2015). E71, m71-m72 [doi:10.1107/S2056989015003710]

\section{Crystal structure of bis $\left(\eta^{5}\right.$-cyclopentadienyl)(1,4-di-tert-butylbuta-1-en-3-yn-1- yl)zirconium(IV) $\mu_{2}$-hydroxido-bis[tris(pentafluorophenyl)borate]}

\section{Vladimir V. Burlakov, Anke Spannenberg, Perdita Arndt and Uwe Rosenthal}

\section{S1. Synthesis and crystallization}

$\mathrm{B}\left(\mathrm{C}_{6} \mathrm{~F}_{5}\right)_{3}(0.376 \mathrm{~g}, 0.73 \mathrm{mmol})$ was dissolved in $20 \mathrm{ml}$ of warm $\left(60^{\circ} \mathrm{C}\right) n$-hexane under Ar, and the obtained solution was added to $\mathrm{Cp}_{2} \mathrm{Zr}\left[(t-\mathrm{Bu}) \mathrm{C}_{4}(t-\mathrm{Bu}](0.282 \mathrm{~g}, 0.73 \mathrm{mmol})\right.$ in $10 \mathrm{ml}$ of $n$-hexane. At once an orange precipitate was formed, which was separated from the mother liquor by filtration, washed with $n$-hexane, and dried in vacuum. Benzene ( $5 \mathrm{ml})$ was added and after one day at RT yellow crystals of the title complex had formed. Yield : $0.187 \mathrm{~g} \mathrm{(18 \% );} \mathrm{m.p.}$ 150-152 ${ }^{\circ} \mathrm{C}$ (dec.) under Ar. Anal. Calcd for $\mathrm{C}_{58} \mathrm{H}_{30} \mathrm{~B}_{2} \mathrm{~F}_{30} \mathrm{OZr}$ : C, 48.86; H, 2.21\%. Found: C, 47.66; H, 2.23\%. ${ }^{1} \mathbf{H}$ NMR $\left(300 \mathrm{MHz}, \mathrm{C}_{6} \mathrm{D}_{6}, 297 \mathrm{~K}\right): \delta=0.84(\mathrm{~s}, 9 \mathrm{H}, t-\mathrm{Bu}) ; 1.25$ (s, 9H, $t$-Bu); $5.63(\mathrm{~s}, 5 \mathrm{H}, \mathrm{Cp}) ; 5.63$ (s, $\left.5 \mathrm{H}, \mathrm{Cp}\right) \mathrm{ppm}$; the signals of $\mathrm{C}=\mathrm{CH}$ and $\mathrm{OH}$ were not detected. ${ }^{13} \mathrm{C}$ NMR $\left(75 \mathrm{MHz}, \mathrm{C}_{6} \mathrm{D}_{6}, 297 \mathrm{~K}\right): \delta=29.0,31.3(t-\mathrm{Bu}) ; 113.7,114.8(\mathrm{Cp})$ ppm; other signals have not been found because of poor solubility. Crystals suitable for $\mathrm{X}$-ray crystal structure analysis were obtained from a saturated $n$-hexane solution at RT after one day.

\section{S2. Refinement}

$\mathrm{H} 1$ and $\mathrm{H} 2$ could be found from the difference Fourier map and were refined freely. All other $\mathrm{H}$ atoms were placed in idealized positions with $\mathrm{d}(\mathrm{C}-\mathrm{H})=0.95 \AA(\mathrm{CH}), 0.98 \AA\left(\mathrm{CH}_{3}\right)$ and refined using a riding model with $U_{\text {iso }}(\mathrm{H})$ fixed at 1.2 $U_{\text {eq }}(\mathrm{C})$ for $\mathrm{CH}$ and $1.5 U_{\text {eq }}(\mathrm{C})$ for $\mathrm{CH}_{3}$. One $t$-butyl group is disordered over two sites with occupancies 0.701 (4):0.299 (4). SADI and DANG instructions were used to improve the geometry of the disordered $t$-butyl group. Additionally, the isotropic displacement parameters of C10A, C11A, C12A, C10B, C11B and C12B were restrained to be equal (SIMU). SADI restraint was also used for the ordered $t$-butyl group and AFIX 56 for the Cp rings. 


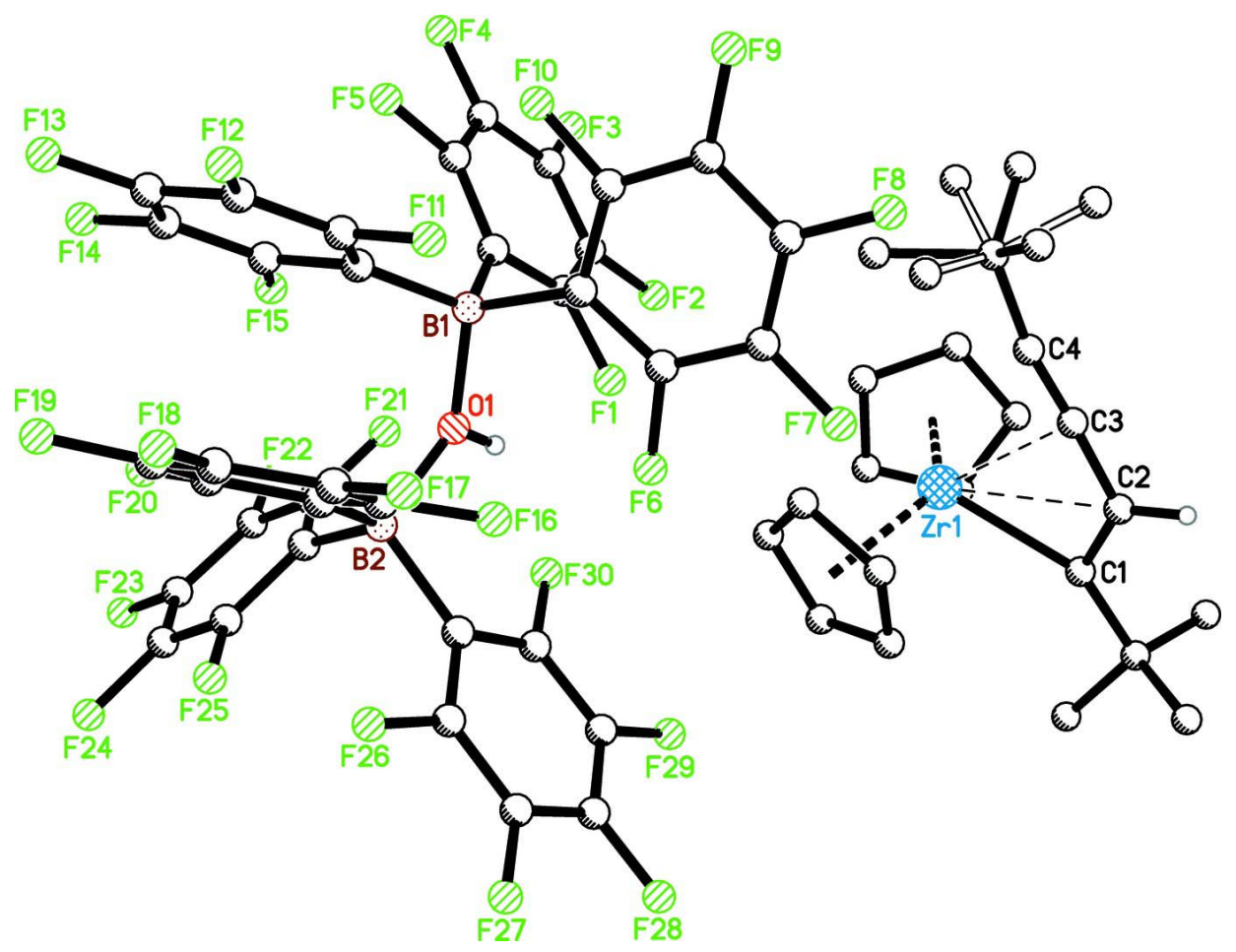

\section{Figure 1}

Ball and stick representation of the molecular structure of the title compound. Zr, B, F, O atoms and $\mathrm{C} 1-\mathrm{C} 4$ are labelled. The minor occupied part of the disordered $t$-butyl group is shown with open lines. Hydrogen atoms of the cyclopentadienyl ligands and the $t$-butyl groups are omitted for clarity. 


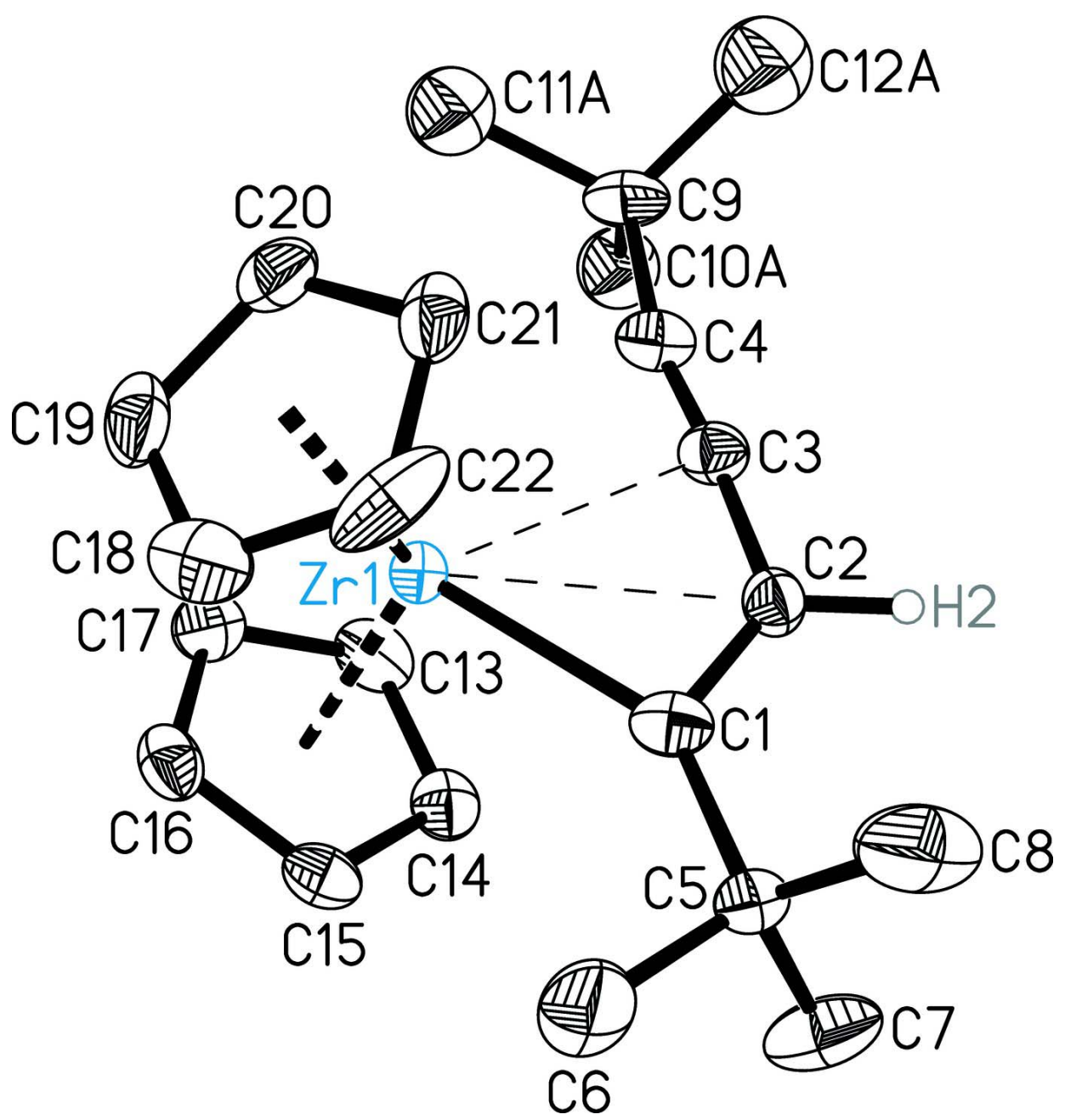

\section{Figure 2}

Molecular structure of the cation with labelling and displacement ellipsoids drawn at 30\% probability level. Hydrogen atoms (except $\mathrm{H} 2$ ) and the minor occupied atoms of the disordered $t$-butyl group are omitted for clarity. 


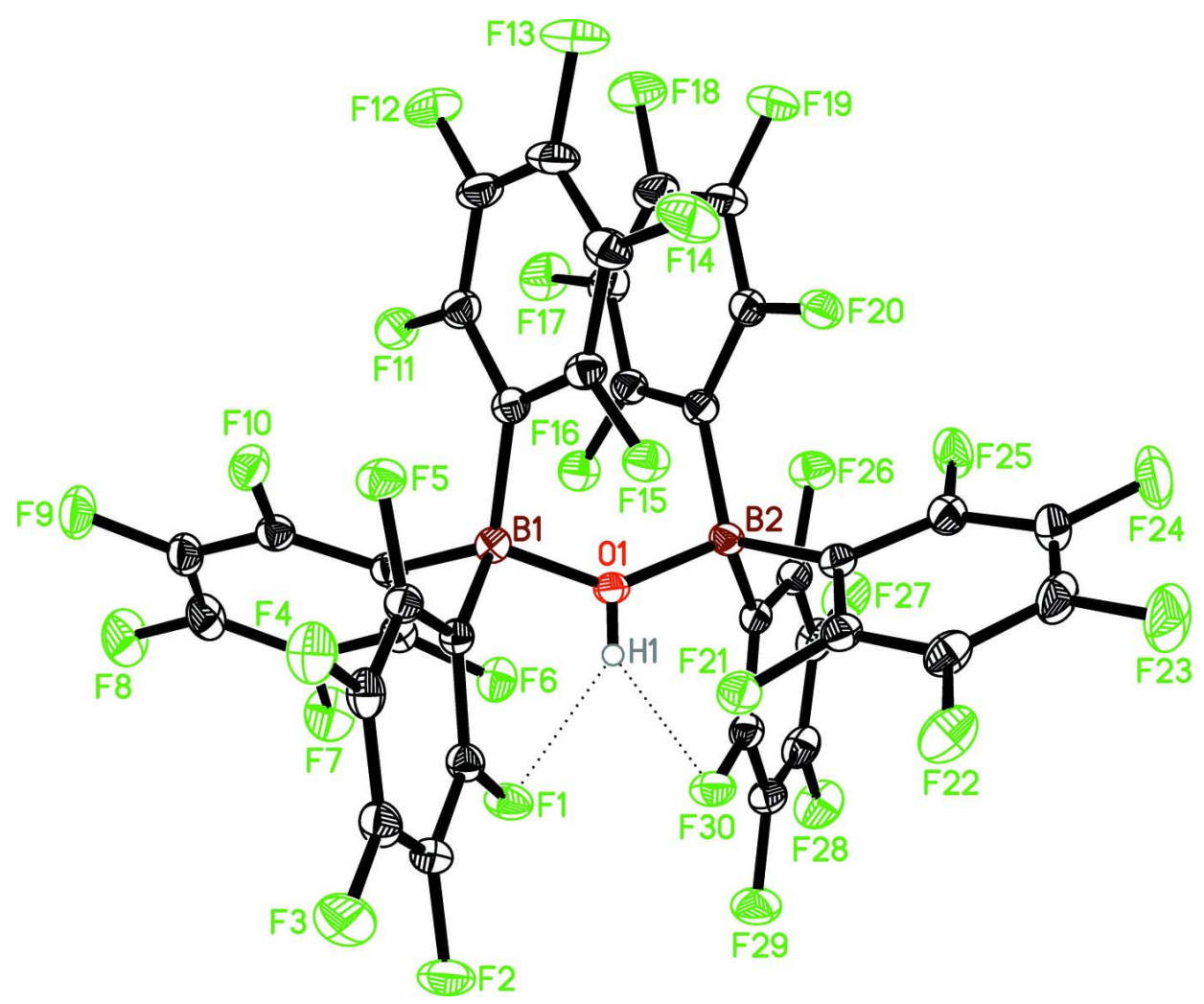

Figure 3

Molecular structure of the anion with labelling of all non-carbon atoms and displacement ellipsoids drawn at 30\% probability level. Hydrogen bonds are shown with dotted lines.

$\operatorname{Bis}\left(\eta^{5}\right.$-cyclopentadienyl)(1,4-di-tert-butylbuta-1-en-3-yn-1-yl)zirconium(IV) $\mu_{2}$-hydroxido-

bis[tris(pentafluorophenyl)borate]

Crystal data

$\left[\mathrm{Zr}\left(\mathrm{C}_{12} \mathrm{H}_{19}\right)\left(\mathrm{C}_{5} \mathrm{H}_{5}\right)_{2}\right]\left(\mathrm{C}_{36} \mathrm{HB}_{2} \mathrm{~F}_{30} \mathrm{O}\right)$

$M_{r}=1425.66$

Triclinic, $P \overline{1}$

$a=12.8896(5) \AA$

$b=13.6334$ (5) $\AA$

$c=16.5466(6) \AA$

$\alpha=86.730(3)^{\circ}$

$\beta=75.389(3)^{\circ}$

$\gamma=77.581(3)^{\circ}$

$V=2747.84(18) \AA^{3}$

$$
\begin{aligned}
& Z=2 \\
& F(000)=1412
\end{aligned}
$$$$
D_{\mathrm{x}}=1.723 \mathrm{Mg} \mathrm{m}^{-3}
$$

Mo $K \alpha$ radiation, $\lambda=0.71073 \AA$

Cell parameters from 6318 reflections

$\theta=1.6-28.4^{\circ}$

$\mu=0.35 \mathrm{~mm}^{-1}$

$T=200 \mathrm{~K}$

Prism, yellow

$0.25 \times 0.25 \times 0.17 \mathrm{~mm}$

\section{Data collection}

Stoe IPDS II

diffractometer

Radiation source: fine-focus sealed tube

Graphite monochromator

$\omega$ scans

Absorption correction: numerical

(X-SHAPE and X-RED32; Stoe \& Cie, 2005)

$T_{\min }=0.810, T_{\max }=0.966$
47172 measured reflections

13119 independent reflections

8407 reflections with $I>2 \sigma(I)$

$R_{\text {int }}=0.040$

$\theta_{\text {max }}=28.0^{\circ}, \theta_{\min }=1.5^{\circ}$

$h=-16 \rightarrow 16$

$k=-17 \rightarrow 17$

$l=-21 \rightarrow 19$ 


\section{Refinement}

Refinement on $F^{2}$

Least-squares matrix: full

$R\left[F^{2}>2 \sigma\left(F^{2}\right)\right]=0.037$

$w R\left(F^{2}\right)=0.083$

$S=0.84$

13119 reflections

820 parameters

27 restraints
Hydrogen site location: mixed

$\mathrm{H}$ atoms treated by a mixture of independent and constrained refinement

$w=1 /\left[\sigma^{2}\left(F_{\mathrm{o}}^{2}\right)+(0.0453 P)^{2}\right]$

where $P=\left(F_{\mathrm{o}}^{2}+2 F_{\mathrm{c}}{ }^{2}\right) / 3$

$(\Delta / \sigma)_{\max }=0.001$

$\Delta \rho_{\max }=0.62 \mathrm{e}^{-3}$

$\Delta \rho_{\min }=-0.41$ e $\AA^{-3}$

Special details

Geometry. All e.s.d.'s (except the e.s.d. in the dihedral angle between two 1.s. planes) are estimated using the full covariance matrix. The cell e.s.d.'s are taken into account individually in the estimation of e.s.d.'s in distances, angles and torsion angles; correlations between e.s.d.'s in cell parameters are only used when they are defined by crystal symmetry. An approximate (isotropic) treatment of cell e.s.d.'s is used for estimating e.s.d.'s involving 1.s. planes.

Fractional atomic coordinates and isotropic or equivalent isotropic displacement parameters $\left(\AA^{2}\right)$

\begin{tabular}{llllll}
\hline & $x$ & $y$ & $z$ & $U_{\text {iso }} * / U_{\text {eq }}$ & Occ. $(<1)$ \\
\hline B1 & $0.62689(19)$ & $0.20536(17)$ & $0.29996(15)$ & $0.0268(5)$ & \\
B2 & $0.79348(19)$ & $0.29432(18)$ & $0.18106(15)$ & $0.0272(5)$ & \\
C1 & $0.9852(2)$ & $0.27187(19)$ & $0.74447(15)$ & $0.0425(6)$ & \\
C2 & $0.9592(2)$ & $0.18318(19)$ & $0.75685(17)$ & $0.0409(6)$ & \\
C3 & $0.8706(2)$ & $0.15060(19)$ & $0.73591(16)$ & $0.0422(6)$ & \\
C4 & $0.7936(2)$ & $0.1297(2)$ & $0.71818(18)$ & $0.0510(7)$ & \\
C5 & $1.0792(2)$ & $0.30077(18)$ & $0.77204(15)$ & $0.0408(6)$ & \\
C6 & $1.0966(3)$ & $0.4026(3)$ & $0.7357(3)$ & $0.0824(12)$ & \\
H6A & 1.1572 & 0.4206 & 0.7534 & $0.124^{*}$ & \\
H6B & 1.0296 & 0.4534 & 0.7557 & $0.124^{*}$ & \\
H6C & 1.1143 & 0.3996 & 0.6746 & $0.124^{*}$ & \\
C7 & $1.0480(3)$ & $0.3083(3)$ & $0.86594(19)$ & $0.0805(11)$ & \\
H7A & 1.0316 & 0.2444 & 0.8902 & $0.121^{*}$ & \\
H7B & 0.9831 & 0.3621 & 0.8835 & $0.121^{*}$ & \\
H7C & 1.1089 & 0.3232 & 0.8851 & $0.121^{*}$ & \\
C8 & $1.1847(3)$ & $0.2256(3)$ & $0.7420(3)$ & $0.1003(15)$ & \\
H8A & 1.2432 & 0.2455 & 0.7610 & $0.150^{*}$ & \\
H8B & 1.2039 & 0.2232 & 0.6808 & $0.150^{*}$ & \\
H8C & 1.1755 & 0.1591 & 0.7646 & $0.150^{*}$ & \\
C9 & $0.7083(2)$ & $0.0823(2)$ & $0.70203(19)$ & $0.0591(8)$ & \\
C10A & $0.6103(4)$ & $0.0946(4)$ & $0.7771(3)$ & $0.0799(14)^{*}$ & $0.701(4)$ \\
H10A & 0.5542 & 0.0628 & 0.7656 & $0.120^{*}$ & $0.701(4)$ \\
H10B & 0.6335 & 0.0628 & 0.8260 & $0.120^{*}$ & $0.701(4)$ \\
H10C & 0.5799 & 0.1663 & 0.7881 & $0.120^{*}$ & $0.701(4)$ \\
C11A & $0.6625(5)$ & $0.1351(4)$ & $0.6285(3)$ & $0.0829(15)^{*}$ & $0.701(4)$ \\
H11A & 0.6061 & 0.1021 & 0.6191 & $0.124^{*}$ & $0.701(4)$ \\
H11B & 0.6305 & 0.2059 & 0.6423 & $0.124^{*}$ & $(4)$ \\
H11C & 0.7222 & 0.1305 & 0.5777 & $0.124^{*}$ & $0.1011(17)^{*}$ \\
C12A & $0.7591(5)$ & $-0.0252(4)$ & $0.6773(4)$ & $0.152^{*}$ & \\
H12A & 0.7035 & -0.0580 & 0.6663 & & \\
& & & & \\
\end{tabular}




\begin{tabular}{|c|c|c|c|c|c|}
\hline H12B & 0.8188 & -0.0271 & 0.6267 & $0.152^{*}$ & $0.701(4)$ \\
\hline $\mathrm{H} 12 \mathrm{C}$ & 0.7883 & -0.0605 & 0.7227 & $0.152 *$ & $0.701(4)$ \\
\hline C10B & $0.5924(7)$ & $0.1386(9)$ & $0.7394(8)$ & $0.083(2)^{*}$ & $0.299(4)$ \\
\hline H10D & 0.5401 & 0.1031 & 0.7263 & $0.124^{*}$ & $0.299(4)$ \\
\hline $\mathrm{H} 10 \mathrm{E}$ & 0.5803 & 0.1426 & 0.8001 & $0.124^{*}$ & 0.299 (4) \\
\hline $\mathrm{H} 10 \mathrm{~F}$ & 0.5819 & 0.2066 & 0.7159 & $0.124 *$ & 0.299 (4) \\
\hline C11B & $0.7348(12)$ & $0.0506(11)$ & $0.6129(5)$ & $0.098(3)^{*}$ & 0.299 (4) \\
\hline H11D & 0.6769 & 0.0194 & 0.6040 & $0.147^{*}$ & $0.299(4)$ \\
\hline H11E & 0.7402 & 0.1095 & 0.5765 & $0.147^{*}$ & $0.299(4)$ \\
\hline $\mathrm{H} 11 \mathrm{~F}$ & 0.8049 & 0.0021 & 0.5998 & $0.147 *$ & $0.299(4)$ \\
\hline C12B & $0.7181(11)$ & $-0.0169(7)$ & $0.7545(8)$ & $0.089(2)^{*}$ & $0.299(4)$ \\
\hline H12D & 0.6637 & -0.0539 & 0.7475 & $0.134^{*}$ & 0.299 (4) \\
\hline $\mathrm{H} 12 \mathrm{E}$ & 0.7918 & -0.0584 & 0.7353 & $0.134^{*}$ & $0.299(4)$ \\
\hline $\mathrm{H} 12 \mathrm{~F}$ & 0.7049 & -0.0005 & 0.8136 & $0.134^{*}$ & $0.299(4)$ \\
\hline C13 & $0.66787(14)$ & $0.36548(12)$ & $0.79695(12)$ & $0.0517(7)$ & \\
\hline H13 & 0.6272 & 0.3145 & 0.8151 & $0.062 *$ & \\
\hline $\mathrm{C} 14$ & $0.74940(15)$ & $0.38772(13)$ & $0.83266(10)$ & $0.0475(7)$ & \\
\hline H14 & 0.7728 & 0.3542 & 0.8789 & $0.057 *$ & \\
\hline $\mathrm{C} 15$ & $0.78976(13)$ & $0.46886(13)$ & $0.78714(12)$ & $0.0479(7)$ & \\
\hline H15 & 0.8449 & 0.4991 & 0.7976 & $0.058^{*}$ & \\
\hline $\mathrm{C} 16$ & $0.73318(15)$ & $0.49676(12)$ & $0.72329(11)$ & $0.0496(7)$ & \\
\hline H16 & 0.7439 & 0.5490 & 0.6835 & $0.060 *$ & \\
\hline $\mathrm{C} 17$ & $0.65784(14)$ & $0.43287(15)$ & 0.72935 (11) & $0.0517(7)$ & \\
\hline H17 & 0.6093 & 0.4349 & 0.6943 & $0.062 *$ & \\
\hline $\mathrm{C} 18$ & $0.9270(2)$ & $0.41749(15)$ & $0.56372(13)$ & $0.0699(10)$ & \\
\hline H18 & 0.9389 & 0.4819 & 0.5730 & $0.084^{*}$ & \\
\hline C19 & $0.83144(17)$ & $0.39720(14)$ & $0.54610(13)$ & $0.0617(8)$ & \\
\hline H19 & 0.7681 & 0.4457 & 0.5415 & $0.074 *$ & \\
\hline $\mathrm{C} 20$ & $0.84714(16)$ & $0.29177(15)$ & $0.53661(13)$ & $0.0552(7)$ & \\
\hline $\mathrm{H} 20$ & 0.7962 & 0.2573 & 0.5245 & $0.066^{*}$ & \\
\hline $\mathrm{C} 21$ & $0.95243(17)$ & $0.24689(14)$ & $0.54838(12)$ & $0.0585(8)$ & \\
\hline $\mathrm{H} 21$ & 0.9842 & 0.1771 & 0.5455 & $0.070^{*}$ & \\
\hline $\mathrm{C} 22$ & $1.00180(14)$ & $0.3246(2)$ & $0.56513(12)$ & $0.0743(11)$ & \\
\hline $\mathrm{H} 22$ & 1.0724 & 0.3159 & 0.5755 & $0.089^{*}$ & \\
\hline $\mathrm{C} 23$ & $0.53417(16)$ & $0.27195(15)$ & $0.37623(13)$ & $0.0259(4)$ & \\
\hline $\mathrm{C} 24$ & $0.56643(17)$ & $0.31399(16)$ & $0.43748(13)$ & $0.0293(5)$ & \\
\hline $\mathrm{C} 25$ & $0.49734(19)$ & $0.36692(17)$ & $0.50575(14)$ & $0.0351(5)$ & \\
\hline $\mathrm{C} 26$ & 0.38647 (19) & $0.37978(17)$ & $0.51604(14)$ & $0.0369(5)$ & \\
\hline $\mathrm{C} 27$ & $0.34887(17)$ & $0.33839(16)$ & $0.45860(14)$ & $0.0328(5)$ & \\
\hline $\mathrm{C} 28$ & $0.42095(17)$ & $0.28547(15)$ & $0.39120(13)$ & $0.0283(5)$ & \\
\hline C29 & $0.67605(17)$ & $0.10434(15)$ & $0.34827(13)$ & $0.0290(5)$ & \\
\hline $\mathrm{C} 30$ & $0.77542(18)$ & $0.08198(16)$ & $0.36996(14)$ & $0.0322(5)$ & \\
\hline C31 & $0.8078(2)$ & $-0.00110(18)$ & $0.41655(16)$ & $0.0416(6)$ & \\
\hline $\mathrm{C} 32$ & $0.7388(2)$ & $-0.06633(17)$ & $0.44457(16)$ & $0.0440(6)$ & \\
\hline C33 & $0.6386(2)$ & $-0.04764(17)$ & $0.42578(15)$ & $0.0394(6)$ & \\
\hline C34 & $0.60956(18)$ & $0.03622(16)$ & $0.37984(14)$ & $0.0314(5)$ & \\
\hline $\mathrm{C} 35$ & $0.57823(16)$ & $0.18643(16)$ & $0.22220(13)$ & $0.0286(5)$ & \\
\hline C36 & $0.59296(18)$ & $0.09457(16)$ & $0.18300(14)$ & $0.0333(5)$ & \\
\hline
\end{tabular}




\begin{tabular}{|c|c|c|c|c|}
\hline C37 & $0.5508(2)$ & $0.08297(19)$ & $0.11677(15)$ & $0.0409(6)$ \\
\hline C38 & $0.4893(2)$ & $0.1647(2)$ & $0.08566(16)$ & $0.0435(6)$ \\
\hline C39 & $0.47201(19)$ & $0.25690(19)$ & $0.12106(15)$ & $0.0388(5)$ \\
\hline $\mathrm{C} 40$ & $0.51676(17)$ & $0.26529(16)$ & $0.18678(14)$ & $0.0306(5)$ \\
\hline $\mathrm{C} 41$ & $0.79519(17)$ & $0.21253(16)$ & $0.11253(13)$ & $0.0292(5)$ \\
\hline $\mathrm{C} 42$ & $0.84955(18)$ & $0.11357(16)$ & $0.11741(14)$ & $0.0324(5)$ \\
\hline $\mathrm{C} 43$ & $0.8606(2)$ & $0.04069(17)$ & $0.06001(16)$ & $0.0400(6)$ \\
\hline $\mathrm{C} 44$ & $0.8142(2)$ & $0.06373(19)$ & $-0.00607(16)$ & $0.0455(6)$ \\
\hline $\mathrm{C} 45$ & $0.7565(2)$ & $0.15938(19)$ & $-0.01305(14)$ & $0.0400(6)$ \\
\hline $\mathrm{C} 46$ & $0.74802(18)$ & $0.23080(16)$ & $0.04529(14)$ & $0.0327(5)$ \\
\hline $\mathrm{C} 47$ & $0.74840(17)$ & $0.41262(16)$ & $0.15988(13)$ & $0.0291(5)$ \\
\hline $\mathrm{C} 48$ & $0.67159(18)$ & $0.48323(16)$ & $0.21216(14)$ & $0.0325(5)$ \\
\hline C49 & 0.64114 (19) & $0.58268(17)$ & $0.19104(17)$ & $0.0398(6)$ \\
\hline C50 & $0.6879(2)$ & $0.61662(17)$ & $0.11353(19)$ & $0.0467(6)$ \\
\hline C51 & $0.7663(2)$ & $0.55051(19)$ & $0.05963(16)$ & $0.0452(6)$ \\
\hline $\mathrm{C} 52$ & $0.79564(19)$ & $0.45291(17)$ & $0.08375(15)$ & $0.0366(5)$ \\
\hline $\mathrm{C} 53$ & $0.91714(17)$ & $0.28909(15)$ & $0.19566(13)$ & $0.0281(4)$ \\
\hline C54 & $1.01596(18)$ & $0.25210(16)$ & $0.13889(14)$ & $0.0335(5)$ \\
\hline C55 & $1.11764(18)$ & $0.24930(17)$ & $0.15358(17)$ & $0.0395(6)$ \\
\hline C56 & $1.12492(19)$ & $0.28574(18)$ & $0.22648(17)$ & $0.0405(6)$ \\
\hline C57 & $1.0302(2)$ & $0.32661(18)$ & $0.28417(16)$ & $0.0385(5)$ \\
\hline C58 & $0.93044(17)$ & $0.32780(16)$ & $0.26670(14)$ & $0.0312(5)$ \\
\hline $\mathrm{F} 1$ & $0.67535(10)$ & $0.30374(11)$ & $0.43197(8)$ & $0.0391(3)$ \\
\hline $\mathrm{F} 2$ & $0.53833(13)$ & $0.40589(12)$ & $0.56093(9)$ & $0.0520(4)$ \\
\hline F3 & $0.31647(13)$ & $0.43285(12)$ & $0.58047(9)$ & $0.0563(4)$ \\
\hline F4 & $0.23980(10)$ & $0.35074(10)$ & $0.46769(9)$ & $0.0447(4)$ \\
\hline F5 & $0.37404(10)$ & $0.24591(10)$ & $0.34052(8)$ & 0.0384 \\
\hline F6 & $0.84787(10)$ & $0.14335(10)$ & $0.34862(9)$ & $0.0401(3)$ \\
\hline F7 & $0.90530(13)$ & $-0.01638(12)$ & $0.43567(11)$ & $0.0603(4)$ \\
\hline F8 & $0.76925(15)$ & $-0.14715(11)$ & $0.48968(11)$ & $0.0651(5)$ \\
\hline F9 & $0.57076(13)$ & -0.11164 (11) & $0.45179(10)$ & $0.0538(4)$ \\
\hline F10 & $0.50912(11)$ & $0.05135(10)$ & $0.36506(9)$ & $0.0403(3)$ \\
\hline F11 & $0.65509(11)$ & $0.01120(9)$ & $0.20768(9)$ & 0.0411 \\
\hline F12 & $0.57139(15)$ & $-0.00692(12)$ & $0.08071(10)$ & $0.0599(4)$ \\
\hline F13 & $0.44963(15)$ & $0.15406(14)$ & $0.02003(10)$ & $0.0652(5)$ \\
\hline F14 & $0.41377(13)$ & $0.33750(12)$ & $0.09076(10)$ & $0.0556(4)$ \\
\hline F15 & $0.49879(11)$ & $0.35877(9)$ & $0.21779(8)$ & $0.0370(3)$ \\
\hline F16 & $0.89746(11)$ & $0.08421(9)$ & $0.18083(9)$ & $0.0406(3)$ \\
\hline F17 & $0.91750(13)$ & $-0.05302(10)$ & $0.06875(10)$ & $0.0570(4)$ \\
\hline F18 & $0.82371(17)$ & $-0.00562(13)$ & $-0.06316(11)$ & $0.0703(5)$ \\
\hline F19 & $0.70551(15)$ & $0.18250(12)$ & $-0.07546(9)$ & $0.0591(4)$ \\
\hline F20 & $0.68685(11)$ & $0.32214(10)$ & $0.03376(8)$ & $0.0402(3)$ \\
\hline F21 & $0.62084(11)$ & $0.45871(9)$ & $0.29049(8)$ & $0.0379(3)$ \\
\hline F22 & $0.56761(12)$ & $0.64748(10)$ & $0.24648(11)$ & $0.0551(4)$ \\
\hline F23 & $0.65937(16)$ & $0.71307(11)$ & $0.09263(12)$ & $0.0713(5)$ \\
\hline F24 & $0.81554(16)$ & $0.58198(12)$ & $-0.01608(11)$ & $0.0698(5)$ \\
\hline F25 & $0.87523(12)$ & $0.39252(10)$ & $0.02851(9)$ & 0.0485 (4) \\
\hline F26 & $1.01811(11)$ & $0.21525(11)$ & $0.06457(8)$ & $0.0436(3)$ \\
\hline
\end{tabular}




\begin{tabular}{lllll} 
F27 & $1.20962(11)$ & $0.21015(11)$ & $0.09655(11)$ & $0.0557(4)$ \\
F28 & $1.22255(12)$ & $0.28281(12)$ & $0.24214(11)$ & $0.0591(4)$ \\
F29 & $1.03531(13)$ & $0.36563(13)$ & $0.35548(10)$ & $0.0558(4)$ \\
F30 & $0.84025(11)$ & $0.37121(10)$ & $0.32578(8)$ & $0.0401(3)$ \\
O1 & $0.71908(12)$ & $0.26558(11)$ & $0.26572(10)$ & $0.0268(3)$ \\
Zr1 & $0.84257(2)$ & $0.32484(2)$ & $0.68602(2)$ & $0.03081(6)$ \\
H1 & $0.733(2)$ & $0.2889(19)$ & $0.3016(16)$ & $0.034(8)^{*}$ \\
H2 & $1.002(3)$ & $0.128(2)$ & $0.782(2)$ & $0.076(10)^{*}$ \\
\hline
\end{tabular}

Atomic displacement parameters $\left(\AA^{2}\right)$

\begin{tabular}{|c|c|c|c|c|c|c|}
\hline & $U^{11}$ & $U^{22}$ & $U^{33}$ & $U^{12}$ & $U^{13}$ & $U^{23}$ \\
\hline B1 & $0.0244(11)$ & $0.0262(11)$ & $0.0289(12)$ & $-0.0044(9)$ & $-0.0053(9)$ & $-0.0019(10)$ \\
\hline B2 & $0.0282(12)$ & $0.0297(12)$ & $0.0230(11)$ & $-0.0063(9)$ & $-0.0046(9)$ & $-0.0017(9)$ \\
\hline $\mathrm{C} 1$ & $0.0434(14)$ & 0.0499 (14) & $0.0320(13)$ & $-0.0175(11)$ & $0.0031(11)$ & $-0.0078(11)$ \\
\hline $\mathrm{C} 2$ & $0.0413(13)$ & $0.0364(13)$ & $0.0460(15)$ & $-0.0085(11)$ & $-0.0133(11)$ & $0.0039(11)$ \\
\hline $\mathrm{C} 3$ & $0.0459(14)$ & $0.0430(14)$ & $0.0399(14)$ & $-0.0163(11)$ & $-0.0089(11)$ & $-0.0001(11)$ \\
\hline $\mathrm{C} 4$ & $0.0512(16)$ & $0.0533(16)$ & $0.0495(16)$ & $-0.0141(13)$ & $-0.0085(13)$ & $-0.0127(13)$ \\
\hline C5 & 0.0407 (13) & $0.0459(14)$ & $0.0378(13)$ & $-0.0111(11)$ & $-0.0106(11)$ & $-0.0049(11)$ \\
\hline C6 & $0.076(2)$ & $0.082(2)$ & $0.116(3)$ & $-0.050(2)$ & $-0.051(2)$ & $0.029(2)$ \\
\hline $\mathrm{C} 7$ & $0.092(3)$ & $0.120(3)$ & $0.0457(18)$ & $-0.055(2)$ & $-0.0184(18)$ & $-0.0064(19)$ \\
\hline $\mathrm{C} 8$ & $0.0468(19)$ & $0.108(3)$ & $0.148(4)$ & $-0.0011(19)$ & $-0.025(2)$ & $-0.065(3)$ \\
\hline C9 & $0.0671(19)$ & $0.0606(18)$ & 0.0601 (19) & $-0.0289(15)$ & $-0.0185(15)$ & $-0.0140(15)$ \\
\hline C13 & $0.0380(14)$ & $0.0508(16)$ & $0.0553(17)$ & $-0.0099(12)$ & $0.0110(12)$ & $-0.0091(13)$ \\
\hline C14 & $0.0481(15)$ & $0.0481(15)$ & $0.0373(14)$ & $-0.0008(12)$ & $-0.0005(12)$ & $-0.0056(12)$ \\
\hline $\mathrm{C} 15$ & $0.0457(14)$ & $0.0425(14)$ & $0.0514(16)$ & $-0.0101(11)$ & $-0.0007(13)$ & $-0.0124(12)$ \\
\hline C16 & $0.0521(15)$ & $0.0378(14)$ & $0.0507(16)$ & $0.0008(12)$ & $-0.0061(13)$ & $-0.0016(12)$ \\
\hline C17 & $0.0350(13)$ & $0.0566(16)$ & $0.0587(18)$ & $0.0051(12)$ & $-0.0119(12)$ & $-0.0148(14)$ \\
\hline $\mathrm{C} 18$ & $0.107(3)$ & $0.068(2)$ & $0.0350(15)$ & $-0.046(2)$ & $0.0052(17)$ & $0.0036(14)$ \\
\hline C19 & $0.074(2)$ & $0.0589(18)$ & $0.0399(15)$ & $0.0079(15)$ & $-0.0128(15)$ & $0.0104(13)$ \\
\hline $\mathrm{C} 20$ & $0.0660(19)$ & $0.0672(19)$ & $0.0375(14)$ & $-0.0172(15)$ & $-0.0198(13)$ & $0.0026(13)$ \\
\hline $\mathrm{C} 21$ & $0.0674(19)$ & $0.0608(18)$ & $0.0311(14)$ & $0.0161(15)$ & $-0.0073(13)$ & $-0.0004(13)$ \\
\hline $\mathrm{C} 22$ & $0.0392(15)$ & $0.161(4)$ & $0.0248(13)$ & $-0.034(2)$ & $-0.0023(12)$ & $0.0045(18)$ \\
\hline $\mathrm{C} 23$ & $0.0281(10)$ & $0.0251(10)$ & $0.0259(10)$ & $-0.0073(8)$ & $-0.0078(8)$ & $0.0020(8)$ \\
\hline C24 & $0.0273(10)$ & $0.0323(11)$ & $0.0275(11)$ & $-0.0065(8)$ & $-0.0047(9)$ & $-0.0009(9)$ \\
\hline $\mathrm{C} 25$ & $0.0433(13)$ & $0.0378(12)$ & $0.0262(11)$ & $-0.0128(10)$ & $-0.0075(10)$ & $-0.0047(9)$ \\
\hline $\mathrm{C} 26$ & $0.0396(13)$ & $0.0321(11)$ & $0.0304(12)$ & $-0.0040(10)$ & $0.0051(10)$ & $-0.0048(9)$ \\
\hline $\mathrm{C} 27$ & $0.0247(10)$ & $0.0315(11)$ & $0.0371(12)$ & $-0.0041(9)$ & $-0.0007(9)$ & $0.0048(9)$ \\
\hline $\mathrm{C} 28$ & $0.0285(10)$ & $0.0284(11)$ & $0.0295(11)$ & $-0.0069(8)$ & $-0.0092(9)$ & $0.0016(9)$ \\
\hline $\mathrm{C} 29$ & $0.0314(11)$ & $0.0277(11)$ & $0.0259(11)$ & $-0.0039(8)$ & $-0.0044(9)$ & $-0.0038(9)$ \\
\hline $\mathrm{C} 30$ & $0.0324(11)$ & $0.0310(11)$ & $0.0317(11)$ & $-0.0044(9)$ & $-0.0068(9)$ & $0.0001(9)$ \\
\hline C31 & $0.0402(13)$ & $0.0392(13)$ & 0.0438 (14) & $0.0023(10)$ & -0.0164 (11) & $0.0015(11)$ \\
\hline $\mathrm{C} 32$ & $0.0561(16)$ & $0.0296(12)$ & $0.0404(14)$ & $0.0006(11)$ & $-0.0114(12)$ & $0.0091(10)$ \\
\hline $\mathrm{C} 33$ & $0.0508(15)$ & $0.0296(11)$ & $0.0340(12)$ & $-0.0104(10)$ & $-0.0021(11)$ & $0.0012(10)$ \\
\hline $\mathrm{C} 34$ & $0.0316(11)$ & $0.0322(11)$ & $0.0296(11)$ & $-0.0057(9)$ & $-0.0060(9)$ & $-0.0036(9)$ \\
\hline $\mathrm{C} 35$ & $0.0259(10)$ & $0.0310(11)$ & $0.0286(11)$ & $-0.0072(8)$ & $-0.0043(9)$ & $-0.0033(9)$ \\
\hline C36 & $0.0358(12)$ & $0.0303(11)$ & $0.0338(12)$ & $-0.0085(9)$ & $-0.0066(10)$ & $-0.0031(9)$ \\
\hline C37 & $0.0457(14)$ & $0.0435(14)$ & $0.0365(13)$ & $-0.0166(11)$ & $-0.0066(11)$ & $-0.0139(11)$ \\
\hline
\end{tabular}




\begin{tabular}{|c|c|c|c|c|c|c|}
\hline C38 & $0.0430(14)$ & $0.0616(17)$ & $0.0336(13)$ & $-0.0150(12)$ & $-0.0178(11)$ & $-0.0094(12)$ \\
\hline C39 & $0.0348(12)$ & $0.0491(14)$ & $0.0335(12)$ & $-0.0054(10)$ & $-0.0133(10)$ & $0.0003(11)$ \\
\hline $\mathrm{C} 40$ & $0.0291(11)$ & $0.0318(11)$ & $0.0308(11)$ & $-0.0061(9)$ & $-0.0062(9)$ & $-0.0052(9)$ \\
\hline C41 & $0.0288(10)$ & $0.0303(11)$ & $0.0270(11)$ & $-0.0066(8)$ & $-0.0027(9)$ & $-0.0035(9)$ \\
\hline $\mathrm{C} 42$ & $0.0332(11)$ & $0.0341(12)$ & $0.0288(11)$ & $-0.0080(9)$ & $-0.0040(9)$ & $-0.0040(9)$ \\
\hline C43 & $0.0400(13)$ & $0.0323(12)$ & $0.0415(14)$ & $-0.0039(10)$ & 0.0005 (11) & $-0.0099(10)$ \\
\hline C44 & $0.0559(16)$ & $0.0429(14)$ & $0.0360(14)$ & $-0.0139(12)$ & $-0.0011(12)$ & $-0.0174(11)$ \\
\hline C45 & $0.0505(14)$ & $0.0475(14)$ & $0.0263(12)$ & $-0.0170(11)$ & $-0.0105(11)$ & $-0.0048(10)$ \\
\hline C46 & $0.0370(12)$ & $0.0319(11)$ & $0.0283(11)$ & $-0.0087(9)$ & $-0.0048(9)$ & $-0.0011(9)$ \\
\hline C47 & $0.0309(11)$ & $0.0293(11)$ & $0.0299(11)$ & $-0.0089(9)$ & $-0.0102(9)$ & $-0.0015(9)$ \\
\hline C48 & $0.0334(11)$ & $0.0311(11)$ & $0.0357(12)$ & $-0.0081(9)$ & $-0.0121(10)$ & $-0.0021(9)$ \\
\hline C49 & $0.0354(12)$ & $0.0297(12)$ & $0.0558(16)$ & $-0.0027(9)$ & $-0.0159(11)$ & -0.0053 \\
\hline C50 & $0.0530(15)$ & $0.0264(12)$ & $0.0660(18)$ & $-0.0074(11)$ & $-0.0272(14)$ & $0.0097(12)$ \\
\hline C51 & $0.0579(16)$ & $0.0403(13)$ & $0.0418(14)$ & $-0.0178(12)$ & $-0.0168(12)$ & $0.0135(11)$ \\
\hline C52 & $0.0402(13)$ & $0.0342(12)$ & $0.0351(12)$ & $-0.0090(10)$ & $-0.0071(10)$ & $-0.0007(10)$ \\
\hline C53 & $0.0307(10)$ & $0.0251(10)$ & $0.0289(11)$ & $-0.0082(8)$ & $-0.0064(9)$ & $0.0007(8)$ \\
\hline C54 & $0.0347(12)$ & $0.0308(11)$ & $0.0345(12)$ & $-0.0091(9)$ & $-0.0052(10)$ & $-0.0017(9)$ \\
\hline C55 & $0.0275(11)$ & $0.0348(12)$ & $0.0523(15)$ & $-0.0063(9)$ & $-0.0029(10)$ & $-0.0013(11)$ \\
\hline C56 & $0.0302(12)$ & $0.0384(13)$ & $0.0593(17)$ & $-0.0121(10)$ & $-0.0196(11)$ & $0.0052(12)$ \\
\hline C57 & $0.0418(13)$ & $0.0424(13)$ & $0.0398(13)$ & $-0.0182(11)$ & $-0.0180(11)$ & $0.0023(11)$ \\
\hline C58 & $0.0305(11)$ & $0.0322(11)$ & $0.0317(12)$ & $-0.0112(9)$ & $-0.0053(9)$ & $-0.0005(9)$ \\
\hline $\mathrm{F} 1$ & $0.0294(7)$ & $0.0595(8)$ & $0.0317(7)$ & $-0.0124(6)$ & $-0.0090(5)$ & $-0.0080(6)$ \\
\hline $\mathrm{F} 2$ & $0.0571(9)$ & $0.0655(10)$ & $0.0360(8)$ & $-0.0182(8)$ & $-0.0071(7)$ & $-0.0207(7)$ \\
\hline F3 & $0.0502(9)$ & $0.0613(10)$ & $0.0441(8)$ & $-0.0036(7)$ & $0.0105(7)$ & $-0.0217(7)$ \\
\hline $\mathrm{F} 4$ & $0.0251(6)$ & $0.0481(8)$ & $0.0534(9)$ & $-0.0038(6)$ & $0.0001(6)$ & $0.0027(7)$ \\
\hline F5 & $0.0288(6)$ & $0.0502(8)$ & $0.0395(7)$ & $-0.0119(6)$ & $-0.0101(6)$ & $-0.0055(6)$ \\
\hline F6 & $0.0315(7)$ & $0.0410(7)$ & $0.0508(8)$ & $-0.0096(6)$ & $-0.0156(6)$ & $0.0071(6)$ \\
\hline F7 & $0.0478(9)$ & $0.0584(10)$ & $0.0765(12)$ & $0.0018(7)$ & $-0.0328(8)$ & $0.0160(8)$ \\
\hline F8 & $0.0813(12)$ & $0.0402(8)$ & $0.0711(11)$ & $-0.0026(8)$ & $-0.0275(9)$ & $0.0239(8)$ \\
\hline F9 & $0.0671(10)$ & $0.0394(8)$ & $0.0545(9)$ & $-0.0230(7)$ & $-0.0066(8)$ & $0.0117(7)$ \\
\hline F10 & $0.0374(7)$ & 0.0407 (7) & $0.0463(8)$ & $-0.0156(6)$ & $-0.0110(6)$ & $0.0044(6)$ \\
\hline F11 & $0.0515(8)$ & $0.0279(7)$ & $0.0414(8)$ & $-0.0040(6)$ & $-0.0095(6)$ & $-0.0060(6)$ \\
\hline F12 & $0.0816(12)$ & $0.0508(9)$ & $0.0539(10)$ & $-0.0201(8)$ & $-0.0184(9)$ & $-0.0239(8)$ \\
\hline F13 & $0.0729(11)$ & $0.0857(12)$ & $0.0507(10)$ & $-0.0167(9)$ & $-0.0363(9)$ & $-0.0176(9)$ \\
\hline F14 & $0.0563(9)$ & $0.0646(10)$ & $0.0487(9)$ & $0.0023(8)$ & $-0.0310(8)$ & $0.0012(8)$ \\
\hline F15 & $0.0427(7)$ & $0.0303(7)$ & $0.0386(7)$ & $-0.0013(6)$ & $-0.0155(6)$ & $-0.0034(6)$ \\
\hline F16 & $0.0434(8)$ & $0.0339(7)$ & $0.0436(8)$ & $-0.0021(6)$ & $-0.0137(6)$ & $-0.0019(6)$ \\
\hline F17 & $0.0633(10)$ & $0.0335(8)$ & $0.0652(11)$ & $0.0039(7)$ & $-0.0079(8)$ & $-0.0169(7)$ \\
\hline F18 & $0.1024(14)$ & $0.0572(10)$ & $0.0512(10)$ & $-0.0145(10)$ & $-0.0137(10)$ & $-0.0310(8)$ \\
\hline F19 & $0.0895(12)$ & $0.0646(10)$ & $0.0352(8)$ & $-0.0253(9)$ & $-0.0287(8)$ & $-0.0044(7)$ \\
\hline F20 & $0.0495(8)$ & $0.0391(7)$ & $0.0352(7)$ & $-0.0069(6)$ & $-0.0183(6)$ & $0.0004(6)$ \\
\hline F21 & $0.0392(7)$ & $0.0352(7)$ & $0.0349(7)$ & $-0.0058(6)$ & $-0.0008(6)$ & $-0.0078(6)$ \\
\hline F22 & $0.0471(8)$ & $0.0335(7)$ & $0.0785(11)$ & $0.0037(6)$ & $-0.0113(8)$ & $-0.0148(7)$ \\
\hline F23 & 0.0904 (13) & $0.0339(8)$ & $0.0910(14)$ & $-0.0052(8)$ & $-0.0359(11)$ & $0.0204(8)$ \\
\hline F24 & 0.0966 (14) & $0.0562(10)$ & $0.0526(10)$ & $-0.0241(9)$ & $-0.0099(9)$ & $0.0259(8)$ \\
\hline F25 & $0.0550(9)$ & $0.0454(8)$ & $0.0354(8)$ & $-0.0113(7)$ & $0.0071(7)$ & $0.0018(6)$ \\
\hline F26 & $0.0355(7)$ & $0.0539(8)$ & $0.0371(8)$ & $-0.0088(6)$ & $0.0011(6)$ & $-0.0134(6)$ \\
\hline F27 & $0.0276(7)$ & $0.0553(9)$ & $0.0757(11)$ & $-0.0061(6)$ & $0.0032(7)$ & $-0.0135(8)$ \\
\hline
\end{tabular}




$\begin{array}{lllllll}\text { F28 } & 0.0360(8) & 0.0666(10) & 0.0839(12) & -0.0153(7) & -0.0278(8) & 0.0012(9) \\ \text { F29 } & 0.0571(9) & 0.0772(11) & 0.0469(9) & -0.0301(8) & -0.0231(8) & -0.0057(8) \\ \text { F30 } & 0.0365(7) & 0.0507(8) & 0.0343(7) & -0.0152(6) & -0.0026(6) & -0.0134(6) \\ \text { O1 } & 0.0281(8) & 0.0298(8) & 0.0243(8) & -0.0091(6) & -0.0065(6) & -0.0033(6) \\ \text { Zr1 } & 0.02742(11) & 0.03248(11) & 0.03073(12) & -0.00538(8) & -0.00494(8) & 0.00140(9)\end{array}$

Geometric parameters $\left(\AA,{ }^{o}\right)$

\begin{tabular}{|c|c|c|c|}
\hline $\mathrm{B} 1-\mathrm{O} 1$ & $1.560(3)$ & $\mathrm{C} 20-\mathrm{C} 21$ & 1.4200 \\
\hline $\mathrm{B} 1-\mathrm{C} 35$ & $1.622(3)$ & $\mathrm{C} 20-\mathrm{Zr} 1$ & $2.522(2)$ \\
\hline $\mathrm{B} 1-\mathrm{C} 29$ & $1.641(3)$ & $\mathrm{C} 20-\mathrm{H} 20$ & 0.9500 \\
\hline $\mathrm{B} 1-\mathrm{C} 23$ & $1.658(3)$ & $\mathrm{C} 21-\mathrm{C} 22$ & 1.4200 \\
\hline $\mathrm{B} 2-\mathrm{O} 1$ & $1.567(3)$ & $\mathrm{C} 21-\mathrm{Zr} 1$ & 2.5147 (19) \\
\hline B2- $-\mathrm{C} 41$ & $1.628(3)$ & $\mathrm{C} 21-\mathrm{H} 21$ & 0.9500 \\
\hline B2-C47 & $1.642(3)$ & $\mathrm{C} 22-\mathrm{Zr} 1$ & $2.4779(18)$ \\
\hline $\mathrm{B} 2-\mathrm{C} 53$ & $1.658(3)$ & $\mathrm{C} 22-\mathrm{H} 22$ & 0.9500 \\
\hline $\mathrm{C} 1-\mathrm{C} 2$ & $1.314(3)$ & $\mathrm{C} 23-\mathrm{C} 24$ & $1.382(3)$ \\
\hline $\mathrm{C} 1-\mathrm{C} 5$ & $1.529(4)$ & $\mathrm{C} 23-\mathrm{C} 28$ & $1.390(3)$ \\
\hline $\mathrm{C} 1-\mathrm{Zr} 1$ & $2.255(3)$ & $\mathrm{C} 24-\mathrm{F} 1$ & $1.361(2)$ \\
\hline $\mathrm{C} 2-\mathrm{C} 3$ & $1.434(4)$ & $\mathrm{C} 24-\mathrm{C} 25$ & $1.378(3)$ \\
\hline $\mathrm{C} 2-\mathrm{Zr} 1$ & $2.597(3)$ & $\mathrm{C} 25-\mathrm{F} 2$ & $1.347(3)$ \\
\hline $\mathrm{C} 2-\mathrm{H} 2$ & $0.96(3)$ & $\mathrm{C} 25-\mathrm{C} 26$ & $1.369(3)$ \\
\hline $\mathrm{C} 3-\mathrm{C} 4$ & $1.197(4)$ & $\mathrm{C} 26-\mathrm{F} 3$ & $1.340(2)$ \\
\hline $\mathrm{C} 3-\mathrm{Zr} 1$ & $2.452(2)$ & $\mathrm{C} 26-\mathrm{C} 27$ & $1.367(4)$ \\
\hline $\mathrm{C} 4-\mathrm{C} 9$ & $1.473(4)$ & $\mathrm{C} 27-\mathrm{F} 4$ & $1.350(2)$ \\
\hline $\mathrm{C} 4-\mathrm{Zr} 1$ & $2.855(3)$ & $\mathrm{C} 27-\mathrm{C} 28$ & $1.381(3)$ \\
\hline $\mathrm{C} 5-\mathrm{C} 8$ & $1.504(4)$ & $\mathrm{C} 28-\mathrm{F} 5$ & $1.344(3)$ \\
\hline $\mathrm{C} 5-\mathrm{C} 7$ & $1.507(4)$ & $\mathrm{C} 29-\mathrm{C} 30$ & $1.383(3)$ \\
\hline $\mathrm{C} 5-\mathrm{C} 6$ & $1.520(4)$ & $\mathrm{C} 29-\mathrm{C} 34$ & $1.390(3)$ \\
\hline C6-H6A & 0.9800 & $\mathrm{C} 30-\mathrm{F} 6$ & $1.354(3)$ \\
\hline C6-H6B & 0.9800 & $\mathrm{C} 30-\mathrm{C} 31$ & $1.382(3)$ \\
\hline C6- $\mathrm{H} 6 \mathrm{C}$ & 0.9800 & $\mathrm{C} 31-\mathrm{F} 7$ & $1.342(3)$ \\
\hline C7-H7A & 0.9800 & $\mathrm{C} 31-\mathrm{C} 32$ & $1.372(4)$ \\
\hline C $7-\mathrm{H} 7 \mathrm{~B}$ & 0.9800 & $\mathrm{C} 32-\mathrm{F} 8$ & $1.339(3)$ \\
\hline $\mathrm{C} 7-\mathrm{H} 7 \mathrm{C}$ & 0.9800 & $\mathrm{C} 32-\mathrm{C} 33$ & $1.371(4)$ \\
\hline $\mathrm{C} 8-\mathrm{H} 8 \mathrm{~A}$ & 0.9800 & C33-F9 & $1.344(3)$ \\
\hline $\mathrm{C} 8-\mathrm{H} 8 \mathrm{~B}$ & 0.9800 & $\mathrm{C} 33-\mathrm{C} 34$ & $1.373(3)$ \\
\hline $\mathrm{C} 8-\mathrm{H} 8 \mathrm{C}$ & 0.9800 & $\mathrm{C} 34-\mathrm{F} 10$ & $1.348(3)$ \\
\hline $\mathrm{C} 9-\mathrm{C} 11 \mathrm{~B}$ & $1.494(7)$ & $\mathrm{C} 35-\mathrm{C} 40$ & $1.387(3)$ \\
\hline $\mathrm{C} 9-\mathrm{C} 12 \mathrm{~A}$ & $1.505(5)$ & $\mathrm{C} 35-\mathrm{C} 36$ & $1.398(3)$ \\
\hline $\mathrm{C} 9-\mathrm{C} 10 \mathrm{~B}$ & $1.516(7)$ & C36-F11 & $1.349(3)$ \\
\hline $\mathrm{C} 9-\mathrm{C} 10 \mathrm{~A}$ & $1.519(5)$ & $\mathrm{C} 36-\mathrm{C} 37$ & $1.370(3)$ \\
\hline $\mathrm{C} 9-\mathrm{C} 11 \mathrm{~A}$ & $1.558(5)$ & C37-F12 & $1.339(3)$ \\
\hline $\mathrm{C} 9-\mathrm{C} 12 \mathrm{~B}$ & $1.564(7)$ & C $37-$ C 38 & $1.377(4)$ \\
\hline $\mathrm{C} 10 \mathrm{~A}-\mathrm{H} 10 \mathrm{~A}$ & 0.9800 & C38-F13 & $1.339(3)$ \\
\hline $\mathrm{C} 10 \mathrm{~A}-\mathrm{H} 10 \mathrm{~B}$ & 0.9800 & $\mathrm{C} 38-\mathrm{C} 39$ & $1.367(4)$ \\
\hline $\mathrm{C} 10 \mathrm{~A}-\mathrm{H} 10 \mathrm{C}$ & 0.9800 & C39-F14 & $1.340(3)$ \\
\hline $\mathrm{C} 11 \mathrm{~A}-\mathrm{H} 11 \mathrm{~A}$ & 0.9800 & $\mathrm{C} 39-\mathrm{C} 40$ & $1.374(3)$ \\
\hline
\end{tabular}




\begin{tabular}{|c|c|c|c|}
\hline $\mathrm{C} 11 \mathrm{~A}-\mathrm{H} 11 \mathrm{~B}$ & 0.9800 & $\mathrm{C} 40-\mathrm{F} 15$ & $1.352(2)$ \\
\hline $\mathrm{C} 11 \mathrm{~A}-\mathrm{H} 11 \mathrm{C}$ & 0.9800 & $\mathrm{C} 41-\mathrm{C} 46$ & $1.384(3)$ \\
\hline $\mathrm{C} 12 \mathrm{~A}-\mathrm{H} 12 \mathrm{~A}$ & 0.9800 & $\mathrm{C} 41-\mathrm{C} 42$ & $1.388(3)$ \\
\hline $\mathrm{C} 12 \mathrm{~A}-\mathrm{H} 12 \mathrm{~B}$ & 0.9800 & $\mathrm{C} 42-\mathrm{F} 16$ & $1.347(3)$ \\
\hline $\mathrm{C} 12 \mathrm{~A}-\mathrm{H} 12 \mathrm{C}$ & 0.9800 & $\mathrm{C} 42-\mathrm{C} 43$ & $1.374(3)$ \\
\hline $\mathrm{C} 10 \mathrm{~B}-\mathrm{H} 10 \mathrm{D}$ & 0.9800 & $\mathrm{C} 43-\mathrm{F} 17$ & $1.349(3)$ \\
\hline $\mathrm{C} 10 \mathrm{~B}-\mathrm{H} 10 \mathrm{E}$ & 0.9800 & $\mathrm{C} 43-\mathrm{C} 44$ & $1.364(4)$ \\
\hline $\mathrm{C} 10 \mathrm{~B}-\mathrm{H} 10 \mathrm{~F}$ & 0.9800 & C44-F18 & $1.340(3)$ \\
\hline $\mathrm{C} 11 \mathrm{~B}-\mathrm{H} 11 \mathrm{D}$ & 0.9800 & $\mathrm{C} 44-\mathrm{C} 45$ & $1.370(4)$ \\
\hline $\mathrm{C} 11 \mathrm{~B}-\mathrm{H} 11 \mathrm{E}$ & 0.9800 & C45-F19 & $1.347(3)$ \\
\hline $\mathrm{C} 11 \mathrm{~B}-\mathrm{H} 11 \mathrm{~F}$ & 0.9800 & $\mathrm{C} 45-\mathrm{C} 46$ & $1.378(3)$ \\
\hline $\mathrm{C} 12 \mathrm{~B}-\mathrm{H} 12 \mathrm{D}$ & 0.9800 & $\mathrm{C} 46-\mathrm{F} 20$ & $1.355(3)$ \\
\hline $\mathrm{C} 12 \mathrm{~B}-\mathrm{H} 12 \mathrm{E}$ & 0.9800 & $\mathrm{C} 47-\mathrm{C} 48$ & $1.381(3)$ \\
\hline $\mathrm{C} 12 \mathrm{~B}-\mathrm{H} 12 \mathrm{~F}$ & 0.9800 & $\mathrm{C} 47-\mathrm{C} 52$ & $1.392(3)$ \\
\hline $\mathrm{C} 13-\mathrm{C} 17$ & 1.4200 & $\mathrm{C} 48-\mathrm{F} 21$ & $1.355(3)$ \\
\hline $\mathrm{C} 13-\mathrm{C} 14$ & 1.4200 & $\mathrm{C} 48-\mathrm{C} 49$ & $1.379(3)$ \\
\hline $\mathrm{C} 13-\mathrm{Zr} 1$ & $2.4982(17)$ & $\mathrm{C} 49-\mathrm{F} 22$ & $1.347(3)$ \\
\hline $\mathrm{C} 13-\mathrm{H} 13$ & 0.9500 & $\mathrm{C} 49-\mathrm{C} 50$ & $1.373(4)$ \\
\hline $\mathrm{C} 14-\mathrm{C} 15$ & 1.4200 & $\mathrm{C} 50-\mathrm{F} 23$ & $1.337(3)$ \\
\hline $\mathrm{C} 14-\mathrm{Zr} 1$ & 2.5277 (17) & $\mathrm{C} 50-\mathrm{C} 51$ & $1.369(4)$ \\
\hline $\mathrm{C} 14-\mathrm{H} 14$ & 0.9500 & $\mathrm{C} 51-\mathrm{F} 24$ & $1.346(3)$ \\
\hline $\mathrm{C} 15-\mathrm{C} 16$ & 1.4200 & C51-C52 & $1.368(3)$ \\
\hline $\mathrm{C} 15-\mathrm{Zr} 1$ & $2.5186(18)$ & C52-F25 & $1.347(3)$ \\
\hline C15-H15 & 0.9500 & $\mathrm{C} 53-\mathrm{C} 58$ & $1.379(3)$ \\
\hline $\mathrm{C} 16-\mathrm{C} 17$ & 1.4200 & $\mathrm{C} 53-\mathrm{C} 54$ & $1.391(3)$ \\
\hline $\mathrm{C} 16-\mathrm{Zr} 1$ & $2.4832(18)$ & $\mathrm{C} 54-\mathrm{F} 26$ & $1.346(3)$ \\
\hline $\mathrm{C} 16-\mathrm{H} 16$ & 0.9500 & C54-C55 & $1.384(3)$ \\
\hline $\mathrm{C} 17-\mathrm{Zr} 1$ & $2.4705(18)$ & $\mathrm{C} 55-\mathrm{F} 27$ & $1.342(3)$ \\
\hline C17-H17 & 0.9500 & $\mathrm{C} 55-\mathrm{C} 56$ & $1.362(4)$ \\
\hline $\mathrm{C} 18-\mathrm{C} 22$ & 1.4200 & C56-F28 & $1.339(3)$ \\
\hline $\mathrm{C} 18-\mathrm{C} 19$ & 1.4200 & $\mathrm{C} 56-\mathrm{C} 57$ & $1.375(4)$ \\
\hline $\mathrm{C} 18-\mathrm{Zr} 1$ & 2.4624 (19) & C57-F29 & $1.343(3)$ \\
\hline C18-H18 & 0.9500 & $\mathrm{C} 57-\mathrm{C} 58$ & $1.384(3)$ \\
\hline $\mathrm{C} 19-\mathrm{C} 20$ & 1.4200 & C58-F30 & $1.362(2)$ \\
\hline C19-Zr1 & $2.4900(19)$ & $\mathrm{O} 1-\mathrm{H} 1$ & $0.76(3)$ \\
\hline C19-H19 & 0.9500 & & \\
\hline $\mathrm{O} 1-\mathrm{B} 1-\mathrm{C} 35$ & $107.31(17)$ & $\mathrm{F} 3-\mathrm{C} 26-\mathrm{C} 27$ & $120.8(2)$ \\
\hline $\mathrm{O} 1-\mathrm{B} 1-\mathrm{C} 29$ & $109.15(17)$ & $\mathrm{F} 3-\mathrm{C} 26-\mathrm{C} 25$ & $120.7(2)$ \\
\hline $\mathrm{C} 35-\mathrm{B} 1-\mathrm{C} 29$ & $115.91(18)$ & $\mathrm{C} 27-\mathrm{C} 26-\mathrm{C} 25$ & $118.5(2)$ \\
\hline $\mathrm{O} 1-\mathrm{B} 1-\mathrm{C} 23$ & $107.78(16)$ & $\mathrm{F} 4-\mathrm{C} 27-\mathrm{C} 26$ & $119.25(19)$ \\
\hline $\mathrm{C} 35-\mathrm{B} 1-\mathrm{C} 23$ & $113.25(17)$ & $\mathrm{F} 4-\mathrm{C} 27-\mathrm{C} 28$ & $119.9(2)$ \\
\hline $\mathrm{C} 29-\mathrm{B} 1-\mathrm{C} 23$ & $103.14(17)$ & $\mathrm{C} 26-\mathrm{C} 27-\mathrm{C} 28$ & $120.8(2)$ \\
\hline $\mathrm{O} 1-\mathrm{B} 2-\mathrm{C} 41$ & $106.53(16)$ & $\mathrm{F} 5-\mathrm{C} 28-\mathrm{C} 27$ & $115.20(19)$ \\
\hline $\mathrm{O} 1-\mathrm{B} 2-\mathrm{C} 47$ & $108.88(16)$ & $\mathrm{F} 5-\mathrm{C} 28-\mathrm{C} 23$ & $121.45(18)$ \\
\hline $\mathrm{C} 41-\mathrm{B} 2-\mathrm{C} 47$ & $116.31(19)$ & $\mathrm{C} 27-\mathrm{C} 28-\mathrm{C} 23$ & $123.3(2)$ \\
\hline $\mathrm{O} 1-\mathrm{B} 2-\mathrm{C} 53$ & $107.65(17)$ & $\mathrm{C} 30-\mathrm{C} 29-\mathrm{C} 34$ & $113.41(19)$ \\
\hline $\mathrm{C} 41-\mathrm{B} 2-\mathrm{C} 53$ & $111.91(17)$ & $\mathrm{C} 30-\mathrm{C} 29-\mathrm{B} 1$ & $127.16(19)$ \\
\hline
\end{tabular}




\begin{tabular}{|c|c|c|c|}
\hline $\mathrm{C} 47-\mathrm{B} 2-\mathrm{C} 53$ & $105.27(17)$ & $\mathrm{C} 34-\mathrm{C} 29-\mathrm{B} 1$ & $119.08(19)$ \\
\hline $\mathrm{C} 2-\mathrm{C} 1-\mathrm{C} 5$ & $124.4(3)$ & $\mathrm{F} 6-\mathrm{C} 30-\mathrm{C} 31$ & $114.6(2)$ \\
\hline $\mathrm{C} 2-\mathrm{C} 1-\mathrm{Zr} 1$ & 89.37 (18) & $\mathrm{F} 6-\mathrm{C} 30-\mathrm{C} 29$ & $121.46(19)$ \\
\hline $\mathrm{C} 5-\mathrm{C} 1-\mathrm{Zr} 1$ & $146.15(18)$ & $\mathrm{C} 31-\mathrm{C} 30-\mathrm{C} 29$ & $123.9(2)$ \\
\hline $\mathrm{C} 1-\mathrm{C} 2-\mathrm{C} 3$ & $128.2(3)$ & $\mathrm{F} 7-\mathrm{C} 31-\mathrm{C} 32$ & $120.4(2)$ \\
\hline $\mathrm{C} 1-\mathrm{C} 2-\mathrm{Zr} 1$ & $60.23(16)$ & $\mathrm{F} 7-\mathrm{C} 31-\mathrm{C} 30$ & $120.0(2)$ \\
\hline $\mathrm{C} 3-\mathrm{C} 2-\mathrm{Zr} 1$ & $68.00(15)$ & $\mathrm{C} 32-\mathrm{C} 31-\mathrm{C} 30$ & $119.6(2)$ \\
\hline $\mathrm{C} 1-\mathrm{C} 2-\mathrm{H} 2$ & $122(2)$ & $\mathrm{F} 8-\mathrm{C} 32-\mathrm{C} 33$ & $120.6(2)$ \\
\hline $\mathrm{C} 3-\mathrm{C} 2-\mathrm{H} 2$ & $109(2)$ & $\mathrm{F} 8-\mathrm{C} 32-\mathrm{C} 31$ & $120.2(2)$ \\
\hline $\mathrm{Zr} 1-\mathrm{C} 2-\mathrm{H} 2$ & $177(2)$ & $\mathrm{C} 33-\mathrm{C} 32-\mathrm{C} 31$ & $119.2(2)$ \\
\hline $\mathrm{C} 4-\mathrm{C} 3-\mathrm{C} 2$ & $175.8(3)$ & $\mathrm{F} 9-\mathrm{C} 33-\mathrm{C} 32$ & $119.9(2)$ \\
\hline $\mathrm{C} 4-\mathrm{C} 3-\mathrm{Zr} 1$ & $96.9(2)$ & $\mathrm{F} 9-\mathrm{C} 33-\mathrm{C} 34$ & $120.9(2)$ \\
\hline $\mathrm{C} 2-\mathrm{C} 3-\mathrm{Zr} 1$ & $79.16(15)$ & $\mathrm{C} 32-\mathrm{C} 33-\mathrm{C} 34$ & $119.2(2)$ \\
\hline $\mathrm{C} 3-\mathrm{C} 4-\mathrm{C} 9$ & $167.8(3)$ & $\mathrm{F} 10-\mathrm{C} 34-\mathrm{C} 33$ & $116.2(2)$ \\
\hline $\mathrm{C} 3-\mathrm{C} 4-\mathrm{Zr} 1$ & $58.49(17)$ & $\mathrm{F} 10-\mathrm{C} 34-\mathrm{C} 29$ & 119.13 (19) \\
\hline $\mathrm{C} 9-\mathrm{C} 4-\mathrm{Zr} 1$ & $133.6(2)$ & $\mathrm{C} 33-\mathrm{C} 34-\mathrm{C} 29$ & $124.6(2)$ \\
\hline $\mathrm{C} 8-\mathrm{C} 5-\mathrm{C} 7$ & $111.1(3)$ & $\mathrm{C} 40-\mathrm{C} 35-\mathrm{C} 36$ & $112.9(2)$ \\
\hline $\mathrm{C} 8-\mathrm{C} 5-\mathrm{C} 6$ & $107.9(3)$ & $\mathrm{C} 40-\mathrm{C} 35-\mathrm{B} 1$ & $120.82(18)$ \\
\hline $\mathrm{C} 7-\mathrm{C} 5-\mathrm{C} 6$ & $108.6(3)$ & $\mathrm{C} 36-\mathrm{C} 35-\mathrm{B} 1$ & $126.22(19)$ \\
\hline $\mathrm{C} 8-\mathrm{C} 5-\mathrm{C} 1$ & $111.2(2)$ & $\mathrm{F} 11-\mathrm{C} 36-\mathrm{C} 37$ & $115.9(2)$ \\
\hline $\mathrm{C} 7-\mathrm{C} 5-\mathrm{C} 1$ & $108.2(2)$ & $\mathrm{F} 11-\mathrm{C} 36-\mathrm{C} 35$ & $120.2(2)$ \\
\hline $\mathrm{C} 6-\mathrm{C} 5-\mathrm{C} 1$ & $109.9(2)$ & $\mathrm{C} 37-\mathrm{C} 36-\mathrm{C} 35$ & $123.8(2)$ \\
\hline $\mathrm{C} 5-\mathrm{C} 6-\mathrm{H} 6 \mathrm{~A}$ & 109.5 & $\mathrm{~F} 12-\mathrm{C} 37-\mathrm{C} 36$ & $120.4(2)$ \\
\hline $\mathrm{C} 5-\mathrm{C} 6-\mathrm{H} 6 \mathrm{~B}$ & 109.5 & $\mathrm{~F} 12-\mathrm{C} 37-\mathrm{C} 38$ & $119.7(2)$ \\
\hline $\mathrm{H} 6 \mathrm{~A}-\mathrm{C} 6-\mathrm{H} 6 \mathrm{~B}$ & 109.5 & $\mathrm{C} 36-\mathrm{C} 37-\mathrm{C} 38$ & $119.9(2)$ \\
\hline $\mathrm{C} 5-\mathrm{C} 6-\mathrm{H} 6 \mathrm{C}$ & 109.5 & $\mathrm{~F} 13-\mathrm{C} 38-\mathrm{C} 39$ & $120.7(2)$ \\
\hline $\mathrm{H} 6 \mathrm{~A}-\mathrm{C} 6-\mathrm{H} 6 \mathrm{C}$ & 109.5 & $\mathrm{~F} 13-\mathrm{C} 38-\mathrm{C} 37$ & $120.0(2)$ \\
\hline $\mathrm{H} 6 \mathrm{~B}-\mathrm{C} 6-\mathrm{H} 6 \mathrm{C}$ & 109.5 & $\mathrm{C} 39-\mathrm{C} 38-\mathrm{C} 37$ & $119.3(2)$ \\
\hline $\mathrm{C} 5-\mathrm{C} 7-\mathrm{H} 7 \mathrm{~A}$ & 109.5 & $\mathrm{~F} 14-\mathrm{C} 39-\mathrm{C} 38$ & $120.0(2)$ \\
\hline $\mathrm{C} 5-\mathrm{C} 7-\mathrm{H} 7 \mathrm{~B}$ & 109.5 & $\mathrm{~F} 14-\mathrm{C} 39-\mathrm{C} 40$ & $121.0(2)$ \\
\hline $\mathrm{H} 7 \mathrm{~A}-\mathrm{C} 7-\mathrm{H} 7 \mathrm{~B}$ & 109.5 & $\mathrm{C} 38-\mathrm{C} 39-\mathrm{C} 40$ & $118.9(2)$ \\
\hline $\mathrm{C} 5-\mathrm{C} 7-\mathrm{H} 7 \mathrm{C}$ & 109.5 & $\mathrm{~F} 15-\mathrm{C} 40-\mathrm{C} 39$ & $116.06(19)$ \\
\hline $\mathrm{H} 7 \mathrm{~A}-\mathrm{C} 7-\mathrm{H} 7 \mathrm{C}$ & 109.5 & $\mathrm{~F} 15-\mathrm{C} 40-\mathrm{C} 35$ & $118.8(2)$ \\
\hline $\mathrm{H} 7 \mathrm{~B}-\mathrm{C} 7-\mathrm{H} 7 \mathrm{C}$ & 109.5 & $\mathrm{C} 39-\mathrm{C} 40-\mathrm{C} 35$ & $125.2(2)$ \\
\hline $\mathrm{C} 5-\mathrm{C} 8-\mathrm{H} 8 \mathrm{~A}$ & 109.5 & $\mathrm{C} 46-\mathrm{C} 41-\mathrm{C} 42$ & $113.3(2)$ \\
\hline $\mathrm{C} 5-\mathrm{C} 8-\mathrm{H} 8 \mathrm{~B}$ & 109.5 & $\mathrm{C} 46-\mathrm{C} 41-\mathrm{B} 2$ & $126.49(19)$ \\
\hline $\mathrm{H} 8 \mathrm{~A}-\mathrm{C} 8-\mathrm{H} 8 \mathrm{~B}$ & 109.5 & $\mathrm{C} 42-\mathrm{C} 41-\mathrm{B} 2$ & $120.2(2)$ \\
\hline $\mathrm{C} 5-\mathrm{C} 8-\mathrm{H} 8 \mathrm{C}$ & 109.5 & $\mathrm{~F} 16-\mathrm{C} 42-\mathrm{C} 43$ & $115.7(2)$ \\
\hline $\mathrm{H} 8 \mathrm{~A}-\mathrm{C} 8-\mathrm{H} 8 \mathrm{C}$ & 109.5 & $\mathrm{~F} 16-\mathrm{C} 42-\mathrm{C} 41$ & $119.9(2)$ \\
\hline $\mathrm{H} 8 \mathrm{~B}-\mathrm{C} 8-\mathrm{H} 8 \mathrm{C}$ & 109.5 & $\mathrm{C} 43-\mathrm{C} 42-\mathrm{C} 41$ & $124.3(2)$ \\
\hline $\mathrm{C} 4-\mathrm{C} 9-\mathrm{C} 11 \mathrm{~B}$ & $111.2(6)$ & $\mathrm{F} 17-\mathrm{C} 43-\mathrm{C} 44$ & $120.2(2)$ \\
\hline $\mathrm{C} 4-\mathrm{C} 9-\mathrm{C} 12 \mathrm{~A}$ & $108.3(3)$ & $\mathrm{F} 17-\mathrm{C} 43-\mathrm{C} 42$ & $120.1(2)$ \\
\hline $\mathrm{C} 4-\mathrm{C} 9-\mathrm{C} 10 \mathrm{~B}$ & $113.8(6)$ & $\mathrm{C} 44-\mathrm{C} 43-\mathrm{C} 42$ & $119.7(2)$ \\
\hline $\mathrm{C} 11 \mathrm{~B}-\mathrm{C} 9-\mathrm{C} 10 \mathrm{~B}$ & $117.1(7)$ & $\mathrm{F} 18-\mathrm{C} 44-\mathrm{C} 43$ & $121.1(2)$ \\
\hline $\mathrm{C} 4-\mathrm{C} 9-\mathrm{C} 10 \mathrm{~A}$ & $110.3(3)$ & $\mathrm{F} 18-\mathrm{C} 44-\mathrm{C} 45$ & $120.1(3)$ \\
\hline $\mathrm{C} 12 \mathrm{~A}-\mathrm{C} 9-\mathrm{C} 10 \mathrm{~A}$ & $114.1(4)$ & $\mathrm{C} 43-\mathrm{C} 44-\mathrm{C} 45$ & $118.9(2)$ \\
\hline $\mathrm{C} 4-\mathrm{C} 9-\mathrm{C} 11 \mathrm{~A}$ & $111.2(3)$ & $\mathrm{F} 19-\mathrm{C} 45-\mathrm{C} 44$ & $119.9(2)$ \\
\hline $\mathrm{C} 12 \mathrm{~A}-\mathrm{C} 9-\mathrm{C} 11 \mathrm{~A}$ & $107.6(4)$ & $\mathrm{F} 19-\mathrm{C} 45-\mathrm{C} 46$ & $120.2(2)$ \\
\hline
\end{tabular}




$\begin{array}{ll}\text { C10A-C9-C11A } & 105.2(4) \\ \text { C4-C9-C12B } & 103.7(6) \\ \text { C11B-C9-C12B } & 106.0(7) \\ \text { C10B-C9-C12B } & 103.5(7) \\ \text { C9-C10A-H10A } & 109.5 \\ \text { C9-C10A-H10B } & 109.5 \\ \text { H10A-C10A-H10B } & 109.5 \\ \text { C9-C10A-H10C } & 109.5 \\ \text { H10A-C10A-H10C } & 109.5 \\ \text { H10B-C10A-H10C } & 109.5 \\ \text { C9-C11A-H11A } & 109.5 \\ \text { C9-C11A-H11B } & 109.5 \\ \text { H11A-C11A-H11B } & 109.5 \\ \text { C9-C11A-H11C } & 109.5 \\ \text { H11A-C11A-H11C } & 109.5 \\ \text { H11B-C11A-H11C } & 109.5 \\ \text { C9-C12A-H12A } & 109.5 \\ \text { C9-C12A-H12B } & 109.5 \\ \text { H12A-C12A-H12B } & 109.5 \\ \text { C9-C12A-H12C } & 109.5 \\ \text { H12A-C12A-H12C } & 109.5 \\ \text { H12B-C12A-H12C } & 109.5 \\ \text { C9-C10B-H10D } & 109.5 \\ \text { C9-C10B-H10E } & 109.5 \\ \text { H10D-C10B-H10E } & 109.5 \\ \text { C9-C10B-H10F } & 109.5 \\ \text { H10D-C10B-H10F } & 109.5 \\ \text { H10E-C10B-H10F } & 109.5 \\ \text { C9-C11B-H11D } & 109.5 \\ \text { C9-C11B-H11E } & 109.5 \\ \text { H11D-C11B-H11E } & 109.5 \\ \text { C9-C11B-H11F } & 109.5 \\ \text { H11D-C11B-H11F } & 109.5 \\ \text { H11E-C11B-H11F } & 109.5 \\ \text { C9-C12B-H12D } & 109.5 \\ \text { C9-C12B-H12E } & 109.5 \\ \text { H12D-C12B-H12E } & 109.5 \\ \text { C9-C12B-H12F } & 109.5 \\ \text { H12D-C12B-H12F } & 109.5 \\ \text { H12E-C12B-H12F } & 109.5 \\ \text { C17-C13-C14 } & 108.0 \\ \text { C17-C13-Zr1 } & 72.33(7) \\ \text { C14-C13-Zr1 } & 74.73(7) \\ \text { C17-C13-H13 } & 126.0 \\ \text { C14-C13-H13 } & 126.0 \\ \text { Zr1-C13-H13 } & 13.8 \\ \text { C15-C14-C13 } & \\ \text { C15-C14-Zr1 } & \\ & \\ & \end{array}$

\begin{tabular}{|c|c|}
\hline $\mathrm{C} 44-\mathrm{C} 45-\mathrm{C} 46$ & $119.9(2)$ \\
\hline $\mathrm{F} 20-\mathrm{C} 46-\mathrm{C} 45$ & $115.1(2)$ \\
\hline $\mathrm{F} 20-\mathrm{C} 46-\mathrm{C} 41$ & $121.0(2)$ \\
\hline $\mathrm{C} 45-\mathrm{C} 46-\mathrm{C} 41$ & $123.9(2)$ \\
\hline $\mathrm{C} 48-\mathrm{C} 47-\mathrm{C} 52$ & $112.9(2)$ \\
\hline $\mathrm{C} 48-\mathrm{C} 47-\mathrm{B} 2$ & $127.54(19)$ \\
\hline $\mathrm{C} 52-\mathrm{C} 47-\mathrm{B} 2$ & 119.34 (19) \\
\hline $\mathrm{F} 21-\mathrm{C} 48-\mathrm{C} 49$ & $114.5(2)$ \\
\hline $\mathrm{F} 21-\mathrm{C} 48-\mathrm{C} 47$ & 121.25 (19) \\
\hline $\mathrm{C} 49-\mathrm{C} 48-\mathrm{C} 47$ & $124.2(2)$ \\
\hline $\mathrm{F} 22-\mathrm{C} 49-\mathrm{C} 50$ & $119.6(2)$ \\
\hline $\mathrm{F} 22-\mathrm{C} 49-\mathrm{C} 48$ & $120.6(2)$ \\
\hline $\mathrm{C} 50-\mathrm{C} 49-\mathrm{C} 48$ & $119.8(2)$ \\
\hline $\mathrm{F} 23-\mathrm{C} 50-\mathrm{C} 51$ & $121.1(2)$ \\
\hline $\mathrm{F} 23-\mathrm{C} 50-\mathrm{C} 49$ & $120.3(2)$ \\
\hline $\mathrm{C} 51-\mathrm{C} 50-\mathrm{C} 49$ & $118.6(2)$ \\
\hline $\mathrm{F} 24-\mathrm{C} 51-\mathrm{C} 52$ & $120.3(2)$ \\
\hline $\mathrm{F} 24-\mathrm{C} 51-\mathrm{C} 50$ & $120.0(2)$ \\
\hline $\mathrm{C} 52-\mathrm{C} 51-\mathrm{C} 50$ & $119.7(2)$ \\
\hline $\mathrm{F} 25-\mathrm{C} 52-\mathrm{C} 51$ & $116.6(2)$ \\
\hline $\mathrm{F} 25-\mathrm{C} 52-\mathrm{C} 47$ & $118.73(19)$ \\
\hline $\mathrm{C} 51-\mathrm{C} 52-\mathrm{C} 47$ & $124.7(2)$ \\
\hline $\mathrm{C} 58-\mathrm{C} 53-\mathrm{C} 54$ & $113.1(2)$ \\
\hline $\mathrm{C} 58-\mathrm{C} 53-\mathrm{B} 2$ & $121.19(18)$ \\
\hline $\mathrm{C} 54-\mathrm{C} 53-\mathrm{B} 2$ & $125.7(2)$ \\
\hline $\mathrm{F} 26-\mathrm{C} 54-\mathrm{C} 55$ & $115.2(2)$ \\
\hline $\mathrm{F} 26-\mathrm{C} 54-\mathrm{C} 53$ & $121.0(2)$ \\
\hline $\mathrm{C} 55-\mathrm{C} 54-\mathrm{C} 53$ & $123.8(2)$ \\
\hline $\mathrm{F} 27-\mathrm{C} 55-\mathrm{C} 56$ & $119.5(2)$ \\
\hline $\mathrm{F} 27-\mathrm{C} 55-\mathrm{C} 54$ & $120.4(2)$ \\
\hline $\mathrm{C} 56-\mathrm{C} 55-\mathrm{C} 54$ & $120.1(2)$ \\
\hline $\mathrm{F} 28-\mathrm{C} 56-\mathrm{C} 55$ & $120.9(2)$ \\
\hline $\mathrm{F} 28-\mathrm{C} 56-\mathrm{C} 57$ & $120.0(2)$ \\
\hline $\mathrm{C} 55-\mathrm{C} 56-\mathrm{C} 57$ & $119.1(2)$ \\
\hline $\mathrm{F} 29-\mathrm{C} 57-\mathrm{C} 56$ & $120.2(2)$ \\
\hline $\mathrm{F} 29-\mathrm{C} 57-\mathrm{C} 58$ & $121.0(2)$ \\
\hline $\mathrm{C} 56-\mathrm{C} 57-\mathrm{C} 58$ & $118.7(2)$ \\
\hline $\mathrm{F} 30-\mathrm{C} 58-\mathrm{C} 53$ & 119.30 (19) \\
\hline $\mathrm{F} 30-\mathrm{C} 58-\mathrm{C} 57$ & $115.6(2)$ \\
\hline $\mathrm{C} 53-\mathrm{C} 58-\mathrm{C} 57$ & $125.1(2)$ \\
\hline $\mathrm{B} 1-\mathrm{O} 1-\mathrm{B} 2$ & $140.58(18)$ \\
\hline $\mathrm{B} 1-\mathrm{O} 1-\mathrm{H} 1$ & $110.4(19)$ \\
\hline $\mathrm{B} 2-\mathrm{O} 1-\mathrm{H} 1$ & $109.0(19)$ \\
\hline $\mathrm{C} 1-\mathrm{Zr} 1-\mathrm{C} 3$ & $63.23(9)$ \\
\hline $\mathrm{C} 1-\mathrm{Zr} 1-\mathrm{C} 18$ & $100.98(9)$ \\
\hline $\mathrm{C} 3-\mathrm{Zr} 1-\mathrm{C} 18$ & $136.21(8)$ \\
\hline $\mathrm{C} 1-\mathrm{Zr} 1-\mathrm{C} 17$ & $135.79(7)$ \\
\hline $\mathrm{C} 3-\mathrm{Zr} 1-\mathrm{C} 17$ & $118.55(8)$ \\
\hline
\end{tabular}




\begin{tabular}{|c|c|c|c|}
\hline $\mathrm{C} 13-\mathrm{C} 14-\mathrm{Zr} 1$ & $72.45(7)$ & $\mathrm{C} 18-\mathrm{Zr} 1-\mathrm{C} 17$ & $101.29(8)$ \\
\hline $\mathrm{C} 15-\mathrm{C} 14-\mathrm{H} 14$ & 126.0 & $\mathrm{C} 1-\mathrm{Zr} 1-\mathrm{C} 22$ & $77.84(7)$ \\
\hline $\mathrm{C} 13-\mathrm{C} 14-\mathrm{H} 14$ & 126.0 & $\mathrm{C} 3-\mathrm{Zr} 1-\mathrm{C} 22$ & $103.64(9)$ \\
\hline $\mathrm{Zr} 1-\mathrm{C} 14-\mathrm{H} 14$ & 120.1 & $\mathrm{C} 18-\mathrm{Zr} 1-\mathrm{C} 22$ & 33.4 \\
\hline $\mathrm{C} 14-\mathrm{C} 15-\mathrm{C} 16$ & 108.0 & $\mathrm{C} 17-\mathrm{Zr} 1-\mathrm{C} 22$ & $134.23(7)$ \\
\hline $\mathrm{C} 14-\mathrm{C} 15-\mathrm{Zr} 1$ & $74.01(6)$ & $\mathrm{C} 1-\mathrm{Zr} 1-\mathrm{C} 16$ & $116.49(8)$ \\
\hline $\mathrm{C} 16-\mathrm{C} 15-\mathrm{Zr} 1$ & $72.14(7)$ & $\mathrm{C} 3-\mathrm{Zr} 1-\mathrm{C} 16$ & $143.21(7)$ \\
\hline $\mathrm{C} 14-\mathrm{C} 15-\mathrm{H} 15$ & 126.0 & $\mathrm{C} 18-\mathrm{Zr} 1-\mathrm{C} 16$ & $80.53(7)$ \\
\hline $\mathrm{C} 16-\mathrm{C} 15-\mathrm{H} 15$ & 126.0 & $\mathrm{C} 17-\mathrm{Zr} 1-\mathrm{C} 16$ & 33.3 \\
\hline $\mathrm{Zr} 1-\mathrm{C} 15-\mathrm{H} 15$ & 119.7 & $\mathrm{C} 22-\mathrm{Zr} 1-\mathrm{C} 16$ & $112.32(8)$ \\
\hline $\mathrm{C} 17-\mathrm{C} 16-\mathrm{C} 15$ & 108.0 & $\mathrm{C} 1-\mathrm{Zr} 1-\mathrm{C} 19$ & $132.07(8)$ \\
\hline $\mathrm{C} 17-\mathrm{C} 16-\mathrm{Zr} 1$ & $72.85(7)$ & $\mathrm{C} 3-\mathrm{Zr} 1-\mathrm{C} 19$ & $131.40(8)$ \\
\hline $\mathrm{C} 15-\mathrm{C} 16-\mathrm{Zr} 1$ & $74.88(7)$ & $\mathrm{C} 18-\mathrm{Zr} 1-\mathrm{C} 19$ & 33.3 \\
\hline $\mathrm{C} 17-\mathrm{C} 16-\mathrm{H} 16$ & 126.0 & $\mathrm{C} 17-\mathrm{Zr} 1-\mathrm{C} 19$ & $82.58(6)$ \\
\hline $\mathrm{C} 15-\mathrm{C} 16-\mathrm{H} 16$ & 126.0 & $\mathrm{C} 22-\mathrm{Zr} 1-\mathrm{C} 19$ & 55.1 \\
\hline $\mathrm{Zr} 1-\mathrm{C} 16-\mathrm{H} 16$ & 118.2 & $\mathrm{C} 16-\mathrm{Zr} 1-\mathrm{C} 19$ & $78.42(6)$ \\
\hline $\mathrm{C} 13-\mathrm{C} 17-\mathrm{C} 16$ & 108.0 & $\mathrm{C} 1-\mathrm{Zr} 1-\mathrm{C} 13$ & $110.14(7)$ \\
\hline $\mathrm{C} 13-\mathrm{C} 17-\mathrm{Zr} 1$ & $74.47(7)$ & $\mathrm{C} 3-\mathrm{Zr} 1-\mathrm{C} 13$ & $89.50(7)$ \\
\hline $\mathrm{C} 16-\mathrm{C} 17-\mathrm{Zr} 1$ & $73.84(7)$ & $\mathrm{C} 18-\mathrm{Zr} 1-\mathrm{C} 13$ & $133.39(7)$ \\
\hline $\mathrm{C} 13-\mathrm{C} 17-\mathrm{H} 17$ & 126.0 & $\mathrm{C} 17-\mathrm{Zr} 1-\mathrm{C} 13$ & 33.2 \\
\hline $\mathrm{C} 16-\mathrm{C} 17-\mathrm{H} 17$ & 126.0 & $\mathrm{C} 22-\mathrm{Zr} 1-\mathrm{C} 13$ & $166.77(8)$ \\
\hline $\mathrm{Zr} 1-\mathrm{C} 17-\mathrm{H} 17$ & 117.7 & $\mathrm{C} 16-\mathrm{Zr} 1-\mathrm{C} 13$ & 54.9 \\
\hline $\mathrm{C} 22-\mathrm{C} 18-\mathrm{C} 19$ & 108.0 & $\mathrm{C} 19-\mathrm{Zr} 1-\mathrm{C} 13$ & $114.67(6)$ \\
\hline $\mathrm{C} 22-\mathrm{C} 18-\mathrm{Zr} 1$ & $73.89(7)$ & $\mathrm{C} 1-\mathrm{Zr} 1-\mathrm{C} 21$ & $91.49(8)$ \\
\hline $\mathrm{C} 19-\mathrm{C} 18-\mathrm{Zr} 1$ & $74.41(8)$ & $\mathrm{C} 3-\mathrm{Zr} 1-\mathrm{C} 21$ & $83.51(7)$ \\
\hline $\mathrm{C} 22-\mathrm{C} 18-\mathrm{H} 18$ & 126.0 & $\mathrm{C} 18-\mathrm{Zr} 1-\mathrm{C} 21$ & 55.0 \\
\hline $\mathrm{C} 19-\mathrm{C} 18-\mathrm{H} 18$ & 126.0 & $\mathrm{C} 17-\mathrm{Zr} 1-\mathrm{C} 21$ & $132.38(6)$ \\
\hline $\mathrm{Zr} 1-\mathrm{C} 18-\mathrm{H} 18$ & 117.7 & $\mathrm{C} 22-\mathrm{Zr} 1-\mathrm{C} 21$ & 33.0 \\
\hline $\mathrm{C} 20-\mathrm{C} 19-\mathrm{C} 18$ & 108.0 & $\mathrm{C} 16-\mathrm{Zr} 1-\mathrm{C} 21$ & $131.87(5)$ \\
\hline $\mathrm{C} 20-\mathrm{C} 19-\mathrm{Zr} 1$ & $74.79(8)$ & $\mathrm{C} 19-\mathrm{Zr} 1-\mathrm{C} 21$ & 54.7 \\
\hline $\mathrm{C} 18-\mathrm{C} 19-\mathrm{Zr} 1$ & $72.28(7)$ & $\mathrm{C} 13-\mathrm{Zr} 1-\mathrm{C} 21$ & $151.26(7)$ \\
\hline $\mathrm{C} 20-\mathrm{C} 19-\mathrm{H} 19$ & 126.0 & $\mathrm{C} 1-\mathrm{Zr} 1-\mathrm{C} 15$ & $85.15(7)$ \\
\hline $\mathrm{C} 18-\mathrm{C} 19-\mathrm{H} 19$ & 126.0 & $\mathrm{C} 3-\mathrm{Zr} 1-\mathrm{C} 15$ & $120.69(7)$ \\
\hline $\mathrm{Zr} 1-\mathrm{C} 19-\mathrm{H} 19$ & 118.8 & $\mathrm{C} 18-\mathrm{Zr} 1-\mathrm{C} 15$ & $96.22(6)$ \\
\hline $\mathrm{C} 19-\mathrm{C} 20-\mathrm{C} 21$ & 108.0 & $\mathrm{C} 17-\mathrm{Zr} 1-\mathrm{C} 15$ & 54.8 \\
\hline $\mathrm{C} 19-\mathrm{C} 20-\mathrm{Zr} 1$ & $72.31(7)$ & $\mathrm{C} 22-\mathrm{Zr} 1-\mathrm{C} 15$ & $117.67(7)$ \\
\hline $\mathrm{C} 21-\mathrm{C} 20-\mathrm{Zr} 1$ & $73.34(7)$ & $\mathrm{C} 16-\mathrm{Zr} 1-\mathrm{C} 15$ & 33.0 \\
\hline $\mathrm{C} 19-\mathrm{C} 20-\mathrm{H} 20$ & 126.0 & $\mathrm{C} 19-\mathrm{Zr} 1-\mathrm{C} 15$ & $107.37(6)$ \\
\hline $\mathrm{C} 21-\mathrm{C} 20-\mathrm{H} 20$ & 126.0 & $\mathrm{C} 13-\mathrm{Zr} 1-\mathrm{C} 15$ & 54.5 \\
\hline $\mathrm{Zr} 1-\mathrm{C} 20-\mathrm{H} 20$ & 120.2 & $\mathrm{C} 21-\mathrm{Zr} 1-\mathrm{C} 15$ & $149.83(7)$ \\
\hline $\mathrm{C} 20-\mathrm{C} 21-\mathrm{C} 22$ & 108.0 & $\mathrm{C} 1-\mathrm{Zr} 1-\mathrm{C} 20$ & $124.24(8)$ \\
\hline $\mathrm{C} 20-\mathrm{C} 21-\mathrm{Zr} 1$ & $73.91(7)$ & $\mathrm{C} 3-\mathrm{Zr} 1-\mathrm{C} 20$ & $98.49(8)$ \\
\hline $\mathrm{C} 22-\mathrm{C} 21-\mathrm{Zr} 1$ & $72.06(7)$ & $\mathrm{C} 18-\mathrm{Zr} 1-\mathrm{C} 20$ & 54.9 \\
\hline $\mathrm{C} 20-\mathrm{C} 21-\mathrm{H} 21$ & 126.0 & $\mathrm{C} 17-\mathrm{Zr} 1-\mathrm{C} 20$ & $99.78(6)$ \\
\hline $\mathrm{C} 22-\mathrm{C} 21-\mathrm{H} 21$ & 126.0 & $\mathrm{C} 22-\mathrm{Zr} 1-\mathrm{C} 20$ & 54.7 \\
\hline $\mathrm{Zr} 1-\mathrm{C} 21-\mathrm{H} 21$ & 119.9 & $\mathrm{C} 16-\mathrm{Zr} 1-\mathrm{C} 20$ & $108.20(6)$ \\
\hline $\mathrm{C} 18-\mathrm{C} 22-\mathrm{C} 21$ & 108.0 & $\mathrm{C} 19-\mathrm{Zr} 1-\mathrm{C} 20$ & 32.9 \\
\hline $\mathrm{C} 18-\mathrm{C} 22-\mathrm{Zr} 1$ & $72.70(7)$ & $\mathrm{C} 13-\mathrm{Zr} 1-\mathrm{C} 20$ & $122.54(7)$ \\
\hline
\end{tabular}




\begin{tabular}{|c|c|c|c|}
\hline $\mathrm{C} 21-\mathrm{C} 22-\mathrm{Zr} 1$ & $74.90(8)$ & $\mathrm{C} 21-\mathrm{Zr} 1-\mathrm{C} 20$ & 32.8 \\
\hline $\mathrm{C} 18-\mathrm{C} 22-\mathrm{H} 22$ & 126.0 & $\mathrm{C} 15-\mathrm{Zr} 1-\mathrm{C} 20$ & $139.66(6)$ \\
\hline $\mathrm{C} 21-\mathrm{C} 22-\mathrm{H} 22$ & 126.0 & $\mathrm{C} 1-\mathrm{Zr} 1-\mathrm{C} 14$ & $81.68(7)$ \\
\hline $\mathrm{Zr} 1-\mathrm{C} 22-\mathrm{H} 22$ & 118.3 & $\mathrm{C} 3-\mathrm{Zr} 1-\mathrm{C} 14$ & $90.97(7)$ \\
\hline $\mathrm{C} 24-\mathrm{C} 23-\mathrm{C} 28$ & $112.72(19)$ & $\mathrm{C} 18-\mathrm{Zr} 1-\mathrm{C} 14$ & $128.86(6)$ \\
\hline $\mathrm{C} 24-\mathrm{C} 23-\mathrm{B} 1$ & $120.32(18)$ & $\mathrm{C} 17-\mathrm{Zr} 1-\mathrm{C} 14$ & 54.7 \\
\hline $\mathrm{C} 28-\mathrm{C} 23-\mathrm{B} 1$ & $126.75(19)$ & $\mathrm{C} 22-\mathrm{Zr} 1-\mathrm{C} 14$ & $145.77(7)$ \\
\hline $\mathrm{F} 1-\mathrm{C} 24-\mathrm{C} 25$ & $115.3(2)$ & $\mathrm{C} 16-\mathrm{Zr} 1-\mathrm{C} 14$ & 54.6 \\
\hline $\mathrm{F} 1-\mathrm{C} 24-\mathrm{C} 23$ & $118.95(18)$ & $\mathrm{C} 19-\mathrm{Zr} 1-\mathrm{C} 14$ & $132.40(5)$ \\
\hline $\mathrm{C} 25-\mathrm{C} 24-\mathrm{C} 23$ & $125.7(2)$ & $\mathrm{C} 13-\mathrm{Zr} 1-\mathrm{C} 14$ & 32.8 \\
\hline $\mathrm{F} 2-\mathrm{C} 25-\mathrm{C} 26$ & $120.7(2)$ & $\mathrm{C} 21-\mathrm{Zr} 1-\mathrm{C} 14$ & $172.66(6)$ \\
\hline $\mathrm{F} 2-\mathrm{C} 25-\mathrm{C} 24$ & $120.5(2)$ & $\mathrm{C} 15-\mathrm{Zr} 1-\mathrm{C} 14$ & 32.7 \\
\hline $\mathrm{C} 26-\mathrm{C} 25-\mathrm{C} 24$ & $118.8(2)$ & $\mathrm{C} 20-\mathrm{Zr} 1-\mathrm{C} 14$ & $153.94(6)$ \\
\hline $\mathrm{C} 5-\mathrm{C} 1-\mathrm{C} 2-\mathrm{C} 3$ & $177.6(2)$ & $\mathrm{B} 1-\mathrm{C} 35-\mathrm{C} 36-\mathrm{F} 11$ & $-1.9(3)$ \\
\hline $\mathrm{Zr} 1-\mathrm{C} 1-\mathrm{C} 2-\mathrm{C} 3$ & $0.0(3)$ & $\mathrm{C} 40-\mathrm{C} 35-\mathrm{C} 36-\mathrm{C} 37$ & $-0.3(3)$ \\
\hline $\mathrm{C} 5-\mathrm{C} 1-\mathrm{C} 2-\mathrm{Zr} 1$ & $177.6(3)$ & $\mathrm{B} 1-\mathrm{C} 35-\mathrm{C} 36-\mathrm{C} 37$ & $-179.3(2)$ \\
\hline $\mathrm{C} 1-\mathrm{C} 2-\mathrm{C} 3-\mathrm{Zr} 1$ & $0.0(3)$ & $\mathrm{F} 11-\mathrm{C} 36-\mathrm{C} 37-\mathrm{F} 12$ & $0.3(3)$ \\
\hline $\mathrm{Zr} 1-\mathrm{C} 3-\mathrm{C} 4-\mathrm{C} 9$ & $174.4(13)$ & $\mathrm{C} 35-\mathrm{C} 36-\mathrm{C} 37-\mathrm{F} 12$ & $177.8(2)$ \\
\hline $\mathrm{C} 2-\mathrm{C} 1-\mathrm{C} 5-\mathrm{C} 8$ & $51.8(4)$ & $\mathrm{F} 11-\mathrm{C} 36-\mathrm{C} 37-\mathrm{C} 38$ & $-178.0(2)$ \\
\hline $\mathrm{Zr} 1-\mathrm{C} 1-\mathrm{C} 5-\mathrm{C} 8$ & $-132.4(3)$ & $\mathrm{C} 35-\mathrm{C} 36-\mathrm{C} 37-\mathrm{C} 38$ & $-0.5(4)$ \\
\hline $\mathrm{C} 2-\mathrm{C} 1-\mathrm{C} 5-\mathrm{C} 7$ & $-70.5(3)$ & $\mathrm{F} 12-\mathrm{C} 37-\mathrm{C} 38-\mathrm{F} 13$ & $0.5(4)$ \\
\hline $\mathrm{Zr} 1-\mathrm{C} 1-\mathrm{C} 5-\mathrm{C} 7$ & $105.3(3)$ & $\mathrm{C} 36-\mathrm{C} 37-\mathrm{C} 38-\mathrm{F} 13$ & $178.8(2)$ \\
\hline $\mathrm{C} 2-\mathrm{C} 1-\mathrm{C} 5-\mathrm{C} 6$ & $171.1(3)$ & $\mathrm{F} 12-\mathrm{C} 37-\mathrm{C} 38-\mathrm{C} 39$ & $-177.7(2)$ \\
\hline $\mathrm{Zr} 1-\mathrm{C} 1-\mathrm{C} 5-\mathrm{C} 6$ & $-13.1(4)$ & $\mathrm{C} 36-\mathrm{C} 37-\mathrm{C} 38-\mathrm{C} 39$ & $0.6(4)$ \\
\hline $\mathrm{C} 3-\mathrm{C} 4-\mathrm{C} 9-\mathrm{C} 11 \mathrm{~B}$ & $-94.7(15)$ & $\mathrm{F} 13-\mathrm{C} 38-\mathrm{C} 39-\mathrm{F} 14$ & $0.8(4)$ \\
\hline $\mathrm{Zr} 1-\mathrm{C} 4-\mathrm{C} 9-\mathrm{C} 11 \mathrm{~B}$ & $78.7(6)$ & $\mathrm{C} 37-\mathrm{C} 38-\mathrm{C} 39-\mathrm{F} 14$ & $179.0(2)$ \\
\hline $\mathrm{C} 3-\mathrm{C} 4-\mathrm{C} 9-\mathrm{C} 12 \mathrm{~A}$ & $-31.3(15)$ & $\mathrm{F} 13-\mathrm{C} 38-\mathrm{C} 39-\mathrm{C} 40$ & $-178.1(2)$ \\
\hline $\mathrm{Zr} 1-\mathrm{C} 4-\mathrm{C} 9-\mathrm{C} 12 \mathrm{~A}$ & $142.1(3)$ & $\mathrm{C} 37-\mathrm{C} 38-\mathrm{C} 39-\mathrm{C} 40$ & $0.1(4)$ \\
\hline $\mathrm{C} 3-\mathrm{C} 4-\mathrm{C} 9-\mathrm{C} 10 \mathrm{~B}$ & $130.5(14)$ & $\mathrm{F} 14-\mathrm{C} 39-\mathrm{C} 40-\mathrm{F} 15$ & $-0.3(3)$ \\
\hline $\mathrm{Zr} 1-\mathrm{C} 4-\mathrm{C} 9-\mathrm{C} 10 \mathrm{~B}$ & $-56.1(7)$ & $\mathrm{C} 38-\mathrm{C} 39-\mathrm{C} 40-\mathrm{F} 15$ & $178.5(2)$ \\
\hline $\mathrm{C} 3-\mathrm{C} 4-\mathrm{C} 9-\mathrm{C} 10 \mathrm{~A}$ & $94.3(14)$ & $\mathrm{F} 14-\mathrm{C} 39-\mathrm{C} 40-\mathrm{C} 35$ & $-179.9(2)$ \\
\hline $\mathrm{Zr} 1-\mathrm{C} 4-\mathrm{C} 9-\mathrm{C} 10 \mathrm{~A}$ & $-92.4(4)$ & $\mathrm{C} 38-\mathrm{C} 39-\mathrm{C} 40-\mathrm{C} 35$ & $-1.0(4)$ \\
\hline $\mathrm{C} 3-\mathrm{C} 4-\mathrm{C} 9-\mathrm{C} 11 \mathrm{~A}$ & $-149.4(13)$ & $\mathrm{C} 36-\mathrm{C} 35-\mathrm{C} 40-\mathrm{F} 15$ & $-178.45(18)$ \\
\hline $\mathrm{Zr} 1-\mathrm{C} 4-\mathrm{C} 9-\mathrm{C} 11 \mathrm{~A}$ & $24.0(4)$ & $\mathrm{B} 1-\mathrm{C} 35-\mathrm{C} 40-\mathrm{F} 15$ & $0.6(3)$ \\
\hline $\mathrm{C} 3-\mathrm{C} 4-\mathrm{C} 9-\mathrm{C} 12 \mathrm{~B}$ & $18.8(15)$ & $\mathrm{C} 36-\mathrm{C} 35-\mathrm{C} 40-\mathrm{C} 39$ & $1.1(3)$ \\
\hline $\mathrm{Zr} 1-\mathrm{C} 4-\mathrm{C} 9-\mathrm{C} 12 \mathrm{~B}$ & $-167.8(5)$ & $\mathrm{B} 1-\mathrm{C} 35-\mathrm{C} 40-\mathrm{C} 39$ & $-179.9(2)$ \\
\hline $\mathrm{C} 17-\mathrm{C} 13-\mathrm{C} 14-\mathrm{C} 15$ & 0.0 & $\mathrm{O} 1-\mathrm{B} 2-\mathrm{C} 41-\mathrm{C} 46$ & $111.3(2)$ \\
\hline $\mathrm{Zr} 1-\mathrm{C} 13-\mathrm{C} 14-\mathrm{C} 15$ & $65.19(7)$ & $\mathrm{C} 47-\mathrm{B} 2-\mathrm{C} 41-\mathrm{C} 46$ & $-10.2(3)$ \\
\hline $\mathrm{C} 17-\mathrm{C} 13-\mathrm{C} 14-\mathrm{Zr} 1$ & $-65.19(7)$ & $\mathrm{C} 53-\mathrm{B} 2-\mathrm{C} 41-\mathrm{C} 46$ & $-131.3(2)$ \\
\hline $\mathrm{C} 13-\mathrm{C} 14-\mathrm{C} 15-\mathrm{C} 16$ & 0.0 & $\mathrm{O} 1-\mathrm{B} 2-\mathrm{C} 41-\mathrm{C} 42$ & $-69.9(2)$ \\
\hline $\mathrm{Zr} 1-\mathrm{C} 14-\mathrm{C} 15-\mathrm{C} 16$ & $64.63(7)$ & $\mathrm{C} 47-\mathrm{B} 2-\mathrm{C} 41-\mathrm{C} 42$ & $168.51(18)$ \\
\hline $\mathrm{C} 13-\mathrm{C} 14-\mathrm{C} 15-\mathrm{Zr} 1$ & $-64.63(7)$ & $\mathrm{C} 53-\mathrm{B} 2-\mathrm{C} 41-\mathrm{C} 42$ & $47.5(2)$ \\
\hline $\mathrm{C} 14-\mathrm{C} 15-\mathrm{C} 16-\mathrm{C} 17$ & 0.0 & $\mathrm{C} 46-\mathrm{C} 41-\mathrm{C} 42-\mathrm{F} 16$ & $-178.54(18)$ \\
\hline $\mathrm{Zr} 1-\mathrm{C} 15-\mathrm{C} 16-\mathrm{C} 17$ & $65.86(6)$ & $\mathrm{B} 2-\mathrm{C} 41-\mathrm{C} 42-\mathrm{F} 16$ & $2.5(3)$ \\
\hline $\mathrm{C} 14-\mathrm{C} 15-\mathrm{C} 16-\mathrm{Zr} 1$ & $-65.86(6)$ & $\mathrm{C} 46-\mathrm{C} 41-\mathrm{C} 42-\mathrm{C} 43$ & $2.7(3)$ \\
\hline $\mathrm{C} 14-\mathrm{C} 13-\mathrm{C} 17-\mathrm{C} 16$ & 0.0 & $\mathrm{~B} 2-\mathrm{C} 41-\mathrm{C} 42-\mathrm{C} 43$ & $-176.3(2)$ \\
\hline $\mathrm{Zr} 1-\mathrm{C} 13-\mathrm{C} 17-\mathrm{C} 16$ & $-66.79(7)$ & $\mathrm{F} 16-\mathrm{C} 42-\mathrm{C} 43-\mathrm{F} 17$ & $-0.6(3)$ \\
\hline
\end{tabular}




\begin{tabular}{|c|c|c|c|}
\hline $\mathrm{C} 14-\mathrm{C} 13-\mathrm{C} 17-\mathrm{Zr} 1$ & $66.79(7)$ & $\mathrm{C} 41-\mathrm{C} 42-\mathrm{C} 43-\mathrm{F} 17$ & $178.2(2)$ \\
\hline $\mathrm{C} 15-\mathrm{C} 16-\mathrm{C} 17-\mathrm{C} 13$ & 0.0 & $\mathrm{~F} 16-\mathrm{C} 42-\mathrm{C} 43-\mathrm{C} 44$ & $179.7(2)$ \\
\hline $\mathrm{Zr} 1-\mathrm{C} 16-\mathrm{C} 17-\mathrm{C} 13$ & $67.21(7)$ & $\mathrm{C} 41-\mathrm{C} 42-\mathrm{C} 43-\mathrm{C} 44$ & $-1.4(4)$ \\
\hline $\mathrm{C} 15-\mathrm{C} 16-\mathrm{C} 17-\mathrm{Zr} 1$ & $-67.21(7)$ & $\mathrm{F} 17-\mathrm{C} 43-\mathrm{C} 44-\mathrm{F} 18$ & $0.3(4)$ \\
\hline $\mathrm{C} 22-\mathrm{C} 18-\mathrm{C} 19-\mathrm{C} 20$ & 0.0 & $\mathrm{C} 42-\mathrm{C} 43-\mathrm{C} 44-\mathrm{F} 18$ & $179.9(2)$ \\
\hline $\mathrm{Zr} 1-\mathrm{C} 18-\mathrm{C} 19-\mathrm{C} 20$ & $-66.83(7)$ & $\mathrm{F} 17-\mathrm{C} 43-\mathrm{C} 44-\mathrm{C} 45$ & $179.7(2)$ \\
\hline $\mathrm{C} 22-\mathrm{C} 18-\mathrm{C} 19-\mathrm{Zr} 1$ & $66.83(7)$ & $\mathrm{C} 42-\mathrm{C} 43-\mathrm{C} 44-\mathrm{C} 45$ & $-0.7(4)$ \\
\hline $\mathrm{C} 18-\mathrm{C} 19-\mathrm{C} 20-\mathrm{C} 21$ & 0.0 & $\mathrm{~F} 18-\mathrm{C} 44-\mathrm{C} 45-\mathrm{F} 19$ & $2.7(4)$ \\
\hline $\mathrm{Zr} 1-\mathrm{C} 19-\mathrm{C} 20-\mathrm{C} 21$ & $-65.16(7)$ & $\mathrm{C} 43-\mathrm{C} 44-\mathrm{C} 45-\mathrm{F} 19$ & $-176.7(2)$ \\
\hline $\mathrm{C} 18-\mathrm{C} 19-\mathrm{C} 20-\mathrm{Zr} 1$ & $65.16(7)$ & $\mathrm{F} 18-\mathrm{C} 44-\mathrm{C} 45-\mathrm{C} 46$ & $-179.2(2)$ \\
\hline $\mathrm{C} 19-\mathrm{C} 20-\mathrm{C} 21-\mathrm{C} 22$ & 0.0 & $\mathrm{C} 43-\mathrm{C} 44-\mathrm{C} 45-\mathrm{C} 46$ & $1.3(4)$ \\
\hline $\mathrm{Zr} 1-\mathrm{C} 20-\mathrm{C} 21-\mathrm{C} 22$ & $-64.48(7)$ & $\mathrm{F} 19-\mathrm{C} 45-\mathrm{C} 46-\mathrm{F} 20$ & $-0.3(3)$ \\
\hline $\mathrm{C} 19-\mathrm{C} 20-\mathrm{C} 21-\mathrm{Zr} 1$ & $64.48(7)$ & $\mathrm{C} 44-\mathrm{C} 45-\mathrm{C} 46-\mathrm{F} 20$ & $-178.4(2)$ \\
\hline $\mathrm{C} 19-\mathrm{C} 18-\mathrm{C} 22-\mathrm{C} 21$ & 0.0 & $\mathrm{~F} 19-\mathrm{C} 45-\mathrm{C} 46-\mathrm{C} 41$ & $178.1(2)$ \\
\hline $\mathrm{Zr} 1-\mathrm{C} 18-\mathrm{C} 22-\mathrm{C} 21$ & $67.17(8)$ & $\mathrm{C} 44-\mathrm{C} 45-\mathrm{C} 46-\mathrm{C} 41$ & $0.1(4)$ \\
\hline $\mathrm{C} 19-\mathrm{C} 18-\mathrm{C} 22-\mathrm{Zr} 1$ & $-67.17(8)$ & $\mathrm{C} 42-\mathrm{C} 41-\mathrm{C} 46-\mathrm{F} 20$ & $176.43(18)$ \\
\hline $\mathrm{C} 20-\mathrm{C} 21-\mathrm{C} 22-\mathrm{C} 18$ & 0.0 & $\mathrm{~B} 2-\mathrm{C} 41-\mathrm{C} 46-\mathrm{F} 20$ & $-4.7(3)$ \\
\hline $\mathrm{Zr} 1-\mathrm{C} 21-\mathrm{C} 22-\mathrm{C} 18$ & $-65.70(7)$ & $\mathrm{C} 42-\mathrm{C} 41-\mathrm{C} 46-\mathrm{C} 45$ & $-2.0(3)$ \\
\hline $\mathrm{C} 20-\mathrm{C} 21-\mathrm{C} 22-\mathrm{Zr} 1$ & $65.70(7)$ & $\mathrm{B} 2-\mathrm{C} 41-\mathrm{C} 46-\mathrm{C} 45$ & $176.9(2)$ \\
\hline $\mathrm{O} 1-\mathrm{B} 1-\mathrm{C} 23-\mathrm{C} 24$ & $-46.7(2)$ & $\mathrm{O} 1-\mathrm{B} 2-\mathrm{C} 47-\mathrm{C} 48$ & $11.0(3)$ \\
\hline $\mathrm{C} 35-\mathrm{B} 1-\mathrm{C} 23-\mathrm{C} 24$ & $-165.22(18)$ & $\mathrm{C} 41-\mathrm{B} 2-\mathrm{C} 47-\mathrm{C} 48$ & $131.4(2)$ \\
\hline $\mathrm{C} 29-\mathrm{B} 1-\mathrm{C} 23-\mathrm{C} 24$ & $68.7(2)$ & $\mathrm{C} 53-\mathrm{B} 2-\mathrm{C} 47-\mathrm{C} 48$ & $-104.1(2)$ \\
\hline $\mathrm{O} 1-\mathrm{B} 1-\mathrm{C} 23-\mathrm{C} 28$ & $139.0(2)$ & $\mathrm{O} 1-\mathrm{B} 2-\mathrm{C} 47-\mathrm{C} 52$ & $-174.3(2)$ \\
\hline $\mathrm{C} 35-\mathrm{B} 1-\mathrm{C} 23-\mathrm{C} 28$ & $20.5(3)$ & $\mathrm{C} 41-\mathrm{B} 2-\mathrm{C} 47-\mathrm{C} 52$ & $-54.0(3)$ \\
\hline $\mathrm{C} 29-\mathrm{B} 1-\mathrm{C} 23-\mathrm{C} 28$ & $-105.6(2)$ & $\mathrm{C} 53-\mathrm{B} 2-\mathrm{C} 47-\mathrm{C} 52$ & $70.5(3)$ \\
\hline $\mathrm{C} 28-\mathrm{C} 23-\mathrm{C} 24-\mathrm{F} 1$ & $178.08(18)$ & $\mathrm{C} 52-\mathrm{C} 47-\mathrm{C} 48-\mathrm{F} 21$ & $-176.8(2)$ \\
\hline $\mathrm{B} 1-\mathrm{C} 23-\mathrm{C} 24-\mathrm{F} 1$ & $3.0(3)$ & $\mathrm{B} 2-\mathrm{C} 47-\mathrm{C} 48-\mathrm{F} 21$ & $-1.9(4)$ \\
\hline $\mathrm{C} 28-\mathrm{C} 23-\mathrm{C} 24-\mathrm{C} 25$ & $-1.9(3)$ & $\mathrm{C} 52-\mathrm{C} 47-\mathrm{C} 48-\mathrm{C} 49$ & $2.2(3)$ \\
\hline $\mathrm{B} 1-\mathrm{C} 23-\mathrm{C} 24-\mathrm{C} 25$ & $-177.0(2)$ & $\mathrm{B} 2-\mathrm{C} 47-\mathrm{C} 48-\mathrm{C} 49$ & $177.1(2)$ \\
\hline $\mathrm{F} 1-\mathrm{C} 24-\mathrm{C} 25-\mathrm{F} 2$ & $1.0(3)$ & $\mathrm{F} 21-\mathrm{C} 48-\mathrm{C} 49-\mathrm{F} 22$ & $0.8(3)$ \\
\hline $\mathrm{C} 23-\mathrm{C} 24-\mathrm{C} 25-\mathrm{F} 2$ & $-179.0(2)$ & $\mathrm{C} 47-\mathrm{C} 48-\mathrm{C} 49-\mathrm{F} 22$ & $-178.2(2)$ \\
\hline $\mathrm{F} 1-\mathrm{C} 24-\mathrm{C} 25-\mathrm{C} 26$ & $-179.50(19)$ & $\mathrm{F} 21-\mathrm{C} 48-\mathrm{C} 49-\mathrm{C} 50$ & $179.3(2)$ \\
\hline $\mathrm{C} 23-\mathrm{C} 24-\mathrm{C} 25-\mathrm{C} 26$ & $0.5(3)$ & $\mathrm{C} 47-\mathrm{C} 48-\mathrm{C} 49-\mathrm{C} 50$ & $0.3(4)$ \\
\hline $\mathrm{F} 2-\mathrm{C} 25-\mathrm{C} 26-\mathrm{F} 3$ & $1.1(3)$ & $\mathrm{F} 22-\mathrm{C} 49-\mathrm{C} 50-\mathrm{F} 23$ & $-1.1(4)$ \\
\hline $\mathrm{C} 24-\mathrm{C} 25-\mathrm{C} 26-\mathrm{F} 3$ & $-178.4(2)$ & $\mathrm{C} 48-\mathrm{C} 49-\mathrm{C} 50-\mathrm{F} 23$ & $-179.6(2)$ \\
\hline $\mathrm{F} 2-\mathrm{C} 25-\mathrm{C} 26-\mathrm{C} 27$ & $-179.7(2)$ & $\mathrm{F} 22-\mathrm{C} 49-\mathrm{C} 50-\mathrm{C} 51$ & $176.9(2)$ \\
\hline $\mathrm{C} 24-\mathrm{C} 25-\mathrm{C} 26-\mathrm{C} 27$ & $0.8(3)$ & $\mathrm{C} 48-\mathrm{C} 49-\mathrm{C} 50-\mathrm{C} 51$ & $-1.6(4)$ \\
\hline $\mathrm{F} 3-\mathrm{C} 26-\mathrm{C} 27-\mathrm{F} 4$ & $-0.6(3)$ & $\mathrm{F} 23-\mathrm{C} 50-\mathrm{C} 51-\mathrm{F} 24$ & $-0.5(4)$ \\
\hline $\mathrm{C} 25-\mathrm{C} 26-\mathrm{C} 27-\mathrm{F} 4$ & $-179.7(2)$ & $\mathrm{C} 49-\mathrm{C} 50-\mathrm{C} 51-\mathrm{F} 24$ & $-178.5(2)$ \\
\hline $\mathrm{F} 3-\mathrm{C} 26-\mathrm{C} 27-\mathrm{C} 28$ & $178.6(2)$ & $\mathrm{F} 23-\mathrm{C} 50-\mathrm{C} 51-\mathrm{C} 52$ & $178.4(3)$ \\
\hline $\mathrm{C} 25-\mathrm{C} 26-\mathrm{C} 27-\mathrm{C} 28$ & $-0.6(3)$ & $\mathrm{C} 49-\mathrm{C} 50-\mathrm{C} 51-\mathrm{C} 52$ & $0.3(4)$ \\
\hline $\mathrm{F} 4-\mathrm{C} 27-\mathrm{C} 28-\mathrm{F} 5$ & $-2.7(3)$ & $\mathrm{F} 24-\mathrm{C} 51-\mathrm{C} 52-\mathrm{F} 25$ & $0.5(4)$ \\
\hline $\mathrm{C} 26-\mathrm{C} 27-\mathrm{C} 28-\mathrm{F} 5$ & $178.11(19)$ & $\mathrm{C} 50-\mathrm{C} 51-\mathrm{C} 52-\mathrm{F} 25$ & $-178.4(2)$ \\
\hline $\mathrm{F} 4-\mathrm{C} 27-\mathrm{C} 28-\mathrm{C} 23$ & $178.12(18)$ & $\mathrm{F} 24-\mathrm{C} 51-\mathrm{C} 52-\mathrm{C} 47$ & $-178.7(2)$ \\
\hline $\mathrm{C} 26-\mathrm{C} 27-\mathrm{C} 28-\mathrm{C} 23$ & $-1.1(3)$ & $\mathrm{C} 50-\mathrm{C} 51-\mathrm{C} 52-\mathrm{C} 47$ & $2.4(4)$ \\
\hline $\mathrm{C} 24-\mathrm{C} 23-\mathrm{C} 28-\mathrm{F} 5$ & $-176.94(18)$ & $\mathrm{C} 48-\mathrm{C} 47-\mathrm{C} 52-\mathrm{F} 25$ & $177.3(2)$ \\
\hline $\mathrm{B} 1-\mathrm{C} 23-\mathrm{C} 28-\mathrm{F} 5$ & $-2.3(3)$ & $\mathrm{B} 2-\mathrm{C} 47-\mathrm{C} 52-\mathrm{F} 25$ & $1.9(3)$ \\
\hline $\mathrm{C} 24-\mathrm{C} 23-\mathrm{C} 28-\mathrm{C} 27$ & $2.2(3)$ & $\mathrm{C} 48-\mathrm{C} 47-\mathrm{C} 52-\mathrm{C} 51$ & $-3.5(4)$ \\
\hline
\end{tabular}




\begin{tabular}{|c|c|c|c|}
\hline $\mathrm{B} 1-\mathrm{C} 23-\mathrm{C} 28-\mathrm{C} 27$ & $176.85(19)$ & $\mathrm{B} 2-\mathrm{C} 47-\mathrm{C} 52-\mathrm{C} 51$ & $-178.9(2)$ \\
\hline $\mathrm{O} 1-\mathrm{B} 1-\mathrm{C} 29-\mathrm{C} 30$ & $10.9(3)$ & $\mathrm{O} 1-\mathrm{B} 2-\mathrm{C} 53-\mathrm{C} 58$ & $-43.2(2)$ \\
\hline $\mathrm{C} 35-\mathrm{B} 1-\mathrm{C} 29-\mathrm{C} 30$ & $132.2(2)$ & $\mathrm{C} 41-\mathrm{B} 2-\mathrm{C} 53-\mathrm{C} 58$ & $-159.96(19)$ \\
\hline $\mathrm{C} 23-\mathrm{B} 1-\mathrm{C} 29-\mathrm{C} 30$ & $-103.5(2)$ & $\mathrm{C} 47-\mathrm{B} 2-\mathrm{C} 53-\mathrm{C} 58$ & $72.8(2)$ \\
\hline $\mathrm{O} 1-\mathrm{B} 1-\mathrm{C} 29-\mathrm{C} 34$ & $-176.40(18)$ & $\mathrm{O} 1-\mathrm{B} 2-\mathrm{C} 53-\mathrm{C} 54$ & $140.4(2)$ \\
\hline $\mathrm{C} 35-\mathrm{B} 1-\mathrm{C} 29-\mathrm{C} 34$ & $-55.1(3)$ & $\mathrm{C} 41-\mathrm{B} 2-\mathrm{C} 53-\mathrm{C} 54$ & $23.6(3)$ \\
\hline $\mathrm{C} 23-\mathrm{B} 1-\mathrm{C} 29-\mathrm{C} 34$ & $69.2(2)$ & $\mathrm{C} 47-\mathrm{B} 2-\mathrm{C} 53-\mathrm{C} 54$ & $-103.6(2)$ \\
\hline $\mathrm{C} 34-\mathrm{C} 29-\mathrm{C} 30-\mathrm{F} 6$ & $-176.21(19)$ & $\mathrm{C} 58-\mathrm{C} 53-\mathrm{C} 54-\mathrm{F} 26$ & $-177.27(19)$ \\
\hline $\mathrm{B} 1-\mathrm{C} 29-\mathrm{C} 30-\mathrm{F} 6$ & $-3.2(3)$ & $\mathrm{B} 2-\mathrm{C} 53-\mathrm{C} 54-\mathrm{F} 26$ & $-0.6(3)$ \\
\hline $\mathrm{C} 34-\mathrm{C} 29-\mathrm{C} 30-\mathrm{C} 31$ & $1.6(3)$ & C58-C53-C54-C55 & $3.2(3)$ \\
\hline $\mathrm{B} 1-\mathrm{C} 29-\mathrm{C} 30-\mathrm{C} 31$ & $174.7(2)$ & $\mathrm{B} 2-\mathrm{C} 53-\mathrm{C} 54-\mathrm{C} 55$ & $179.9(2)$ \\
\hline $\mathrm{F} 6-\mathrm{C} 30-\mathrm{C} 31-\mathrm{F} 7$ & $-1.2(3)$ & $\mathrm{F} 26-\mathrm{C} 54-\mathrm{C} 55-\mathrm{F} 27$ & $-1.2(3)$ \\
\hline $\mathrm{C} 29-\mathrm{C} 30-\mathrm{C} 31-\mathrm{F} 7$ & $-179.2(2)$ & $\mathrm{C} 53-\mathrm{C} 54-\mathrm{C} 55-\mathrm{F} 27$ & $178.3(2)$ \\
\hline $\mathrm{F} 6-\mathrm{C} 30-\mathrm{C} 31-\mathrm{C} 32$ & $177.4(2)$ & F26-C54-C55-C56 & $179.3(2)$ \\
\hline $\mathrm{C} 29-\mathrm{C} 30-\mathrm{C} 31-\mathrm{C} 32$ & $-0.6(4)$ & $\mathrm{C} 53-\mathrm{C} 54-\mathrm{C} 55-\mathrm{C} 56$ & $-1.2(4)$ \\
\hline $\mathrm{F} 7-\mathrm{C} 31-\mathrm{C} 32-\mathrm{F} 8$ & $-1.5(4)$ & F27-C55-C56-F28 & $-0.2(3)$ \\
\hline $\mathrm{C} 30-\mathrm{C} 31-\mathrm{C} 32-\mathrm{F} 8$ & $179.9(2)$ & $\mathrm{C} 54-\mathrm{C} 55-\mathrm{C} 56-\mathrm{F} 28$ & $179.4(2)$ \\
\hline $\mathrm{F} 7-\mathrm{C} 31-\mathrm{C} 32-\mathrm{C} 33$ & $178.5(2)$ & $\mathrm{F} 27-\mathrm{C} 55-\mathrm{C} 56-\mathrm{C} 57$ & $179.4(2)$ \\
\hline $\mathrm{C} 30-\mathrm{C} 31-\mathrm{C} 32-\mathrm{C} 33$ & $-0.1(4)$ & $\mathrm{C} 54-\mathrm{C} 55-\mathrm{C} 56-\mathrm{C} 57$ & $-1.1(4)$ \\
\hline $\mathrm{F} 8-\mathrm{C} 32-\mathrm{C} 33-\mathrm{F} 9$ & $-0.9(4)$ & $\mathrm{F} 28-\mathrm{C} 56-\mathrm{C} 57-\mathrm{F} 29$ & $1.4(3)$ \\
\hline $\mathrm{C} 31-\mathrm{C} 32-\mathrm{C} 33-\mathrm{F} 9$ & $179.1(2)$ & $\mathrm{C} 55-\mathrm{C} 56-\mathrm{C} 57-\mathrm{F} 29$ & $-178.1(2)$ \\
\hline $\mathrm{F} 8-\mathrm{C} 32-\mathrm{C} 33-\mathrm{C} 34$ & $179.6(2)$ & $\mathrm{F} 28-\mathrm{C} 56-\mathrm{C} 57-\mathrm{C} 58$ & $-179.4(2)$ \\
\hline $\mathrm{C} 31-\mathrm{C} 32-\mathrm{C} 33-\mathrm{C} 34$ & $-0.4(4)$ & $\mathrm{C} 55-\mathrm{C} 56-\mathrm{C} 57-\mathrm{C} 58$ & $1.1(3)$ \\
\hline $\mathrm{F} 9-\mathrm{C} 33-\mathrm{C} 34-\mathrm{F} 10$ & $1.9(3)$ & $\mathrm{C} 54-\mathrm{C} 53-\mathrm{C} 58-\mathrm{F} 30$ & $176.71(18)$ \\
\hline $\mathrm{C} 32-\mathrm{C} 33-\mathrm{C} 34-\mathrm{F} 10$ & $-178.6(2)$ & $\mathrm{B} 2-\mathrm{C} 53-\mathrm{C} 58-\mathrm{F} 30$ & $-0.1(3)$ \\
\hline $\mathrm{F} 9-\mathrm{C} 33-\mathrm{C} 34-\mathrm{C} 29$ & $-177.8(2)$ & $\mathrm{C} 54-\mathrm{C} 53-\mathrm{C} 58-\mathrm{C} 57$ & $-3.3(3)$ \\
\hline $\mathrm{C} 32-\mathrm{C} 33-\mathrm{C} 34-\mathrm{C} 29$ & $1.6(4)$ & $\mathrm{B} 2-\mathrm{C} 53-\mathrm{C} 58-\mathrm{C} 57$ & $179.9(2)$ \\
\hline $\mathrm{C} 30-\mathrm{C} 29-\mathrm{C} 34-\mathrm{F} 10$ & $178.12(19)$ & $\mathrm{F} 29-\mathrm{C} 57-\mathrm{C} 58-\mathrm{F} 30$ & $0.5(3)$ \\
\hline $\mathrm{B} 1-\mathrm{C} 29-\mathrm{C} 34-\mathrm{F} 10$ & $4.5(3)$ & $\mathrm{C} 56-\mathrm{C} 57-\mathrm{C} 58-\mathrm{F} 30$ & $-178.7(2)$ \\
\hline $\mathrm{C} 30-\mathrm{C} 29-\mathrm{C} 34-\mathrm{C} 33$ & $-2.2(3)$ & $\mathrm{F} 29-\mathrm{C} 57-\mathrm{C} 58-\mathrm{C} 53$ & $-179.5(2)$ \\
\hline $\mathrm{B} 1-\mathrm{C} 29-\mathrm{C} 34-\mathrm{C} 33$ & $-175.8(2)$ & $\mathrm{C} 56-\mathrm{C} 57-\mathrm{C} 58-\mathrm{C} 53$ & $1.3(4)$ \\
\hline $\mathrm{O} 1-\mathrm{B} 1-\mathrm{C} 35-\mathrm{C} 40$ & $-69.8(2)$ & $\mathrm{C} 35-\mathrm{B} 1-\mathrm{O} 1-\mathrm{B} 2$ & $-16.1(3)$ \\
\hline $\mathrm{C} 29-\mathrm{B} 1-\mathrm{C} 35-\mathrm{C} 40$ & $167.98(18)$ & $\mathrm{C} 29-\mathrm{B} 1-\mathrm{O} 1-\mathrm{B} 2$ & $110.2(3)$ \\
\hline $\mathrm{C} 23-\mathrm{B} 1-\mathrm{C} 35-\mathrm{C} 40$ & $49.0(3)$ & $\mathrm{C} 23-\mathrm{B} 1-\mathrm{O} 1-\mathrm{B} 2$ & $-138.4(2)$ \\
\hline $\mathrm{O} 1-\mathrm{B} 1-\mathrm{C} 35-\mathrm{C} 36$ & $109.1(2)$ & $\mathrm{C} 41-\mathrm{B} 2-\mathrm{O} 1-\mathrm{B} 1$ & $-20.7(3)$ \\
\hline $\mathrm{C} 29-\mathrm{B} 1-\mathrm{C} 35-\mathrm{C} 36$ & $-13.1(3)$ & $\mathrm{C} 47-\mathrm{B} 2-\mathrm{O} 1-\mathrm{B} 1$ & $105.5(3)$ \\
\hline $\mathrm{C} 23-\mathrm{B} 1-\mathrm{C} 35-\mathrm{C} 36$ & $-132.1(2)$ & $\mathrm{C} 53-\mathrm{B} 2-\mathrm{O} 1-\mathrm{B} 1$ & $-140.9(2)$ \\
\hline $\mathrm{C} 40-\mathrm{C} 35-\mathrm{C} 36-\mathrm{F} 11$ & $177.04(18)$ & & \\
\hline
\end{tabular}

Hydrogen-bond geometry $\left(A,{ }^{\circ}\right)$

\begin{tabular}{lllll}
\hline$D-\mathrm{H} \cdots A$ & $D-\mathrm{H}$ & $\mathrm{H} \cdots A$ & $D \cdots A$ & $D-\mathrm{H} \cdots A$ \\
\hline $\mathrm{O} 1-\mathrm{H} 1 \cdots \mathrm{F} 1$ & $0.76(3)$ & $2.10(3)$ & $2.722(2)$ & $139(2)$ \\
$\mathrm{O} 1-\mathrm{H} 1 \cdots \mathrm{F} 30$ & $0.76(3)$ & $2.08(3)$ & $2.723(2)$ & $142(2)$ \\
\hline
\end{tabular}

